\title{
Capacity Analysis and Pilot-Data Power Allocation for MIMO-OFDM With Transmitter and Receiver IQ Imbalances and Residual Carrier Frequency Offset
}

\author{
V. K. Varma Gottumukkala and Hlaing Minn, Senior Member, IEEE
}

\begin{abstract}
We analyze the ergodic capacity and capacity bound of a multiple-input-multiple-output (MIMO) orthogonal frequency-division multiplexing (OFDM) system suffering from frequency-selective in-phase and quadrature-phase (IQ) imbalances along with residual carrier frequency offset (CFO). In the literature, capacity analysis of systems with IQ imbalance has been done only for the single antenna case and has considered either frequency-flat IQ imbalances at both transmitter and receiver sides without CFO or only receiver-side IQ imbalances with CFO. Additionally, these works dealt with IQ imbalance by treating the mirror tone interference as an additional source of noise, which is not optimal. In this paper, we consider a generalized system model that includes a multiantenna system with frequencyselective IQ imbalances at both the transmitter and the receiver sides, in addition to the presence of CFO, and perform capacity analysis considering joint detection of the signal and its selfinterference. In addition, for the imperfect channel estimation case, existing works in the literature optimize for pilot spacings and pilot designs. Pilot-data power allocation is an additional degree of freedom that is available to further optimize the system and has not been addressed before. Therefore, we analyze the pilot-data power allocation that maximizes the capacity bound and obtain a closed-form solution for the optimal power allocation at high signal-to-noise ratio (SNR) regime. We then study how the IQ imbalance and residual CFO severity affect capacity, symbol error rate (SER), and capacity bound maximizing power allocation. Finally, we derive the optimal power allocation when the channel is slow varying and remains constant over a block of several OFDM symbols. The derived results are validated through Monte Carlo simulations.
\end{abstract}

Index Terms-Carrier frequency offset (CFO), channel estimation, correlated multiple input-multiple output (MIMO), in-phase and quadrature-phase (IQ) imbalance, orthogonal frequencydivision multiplexing (OFDM), pilot, power allocation.

\section{INTRODUCTION}

$\mathbf{T}$ HE AMPLITUDE and phase mismatch between the inphase and quadrature-phase (IQ) branches of a communication system (termed IQ imbalance) is an impairment arising due to device imperfections, and recently, it has received significant research attention. Without compensation, it can lead to overall system performance degradation.

Manuscript received June 5, 2011; revised October 5, 2011; accepted November 14, 2011. Date of publication December 16, 2011; date of current version February 21, 2012. This paper was presented in part at the IEEE Globecom, Honolulu, HI, December 2009. The review of this paper was coordinated by Prof. C. P. Oestges.

The authors are with the Department of Electrical Engineering, University of Texas at Dallas, Richardson, TX 75080 USA (e-mail: vkg071000@ utdallas.edu; Hlaing.Minn@utdallas.edu).

Digital Object Identifier 10.1109/TVT.2011.2180030
There has been little work in the literature on capacity analysis for systems with IQ imbalance. To the best of our knowledge, [2]-[5] are the only works that consider capacity analysis of an orthogonal frequency-division multiplexing (OFDM) system with IQ imbalance. These works consider a single-input-single-output (SISO) system and treat the selfinterference of the signal due to IQ imbalance as a source of noise in the capacity analysis, which is obviously not optimal, and CFO has been considered only for the case of receiver side only IQ imbalances. Additionally, these works considered only frequency-flat IQ imbalances. In the current work, we consider a generalized system that includes a multiple-input-multipleoutput (MIMO) system with frequency-selective IQ imbalances at both transmitter and receiver sides, in addition to impairment due to $\mathrm{CFO}$ and analyze the capacity by considering joint detection of the signal and its self-interference.

In addition to IQ imbalance, imperfect channel estimation also affects capacity and symbol error rate (SER)/bit error rate (BER) [6]. The existing works on IQ imbalances in the literature under imperfect channel estimation discuss pilot designs [7]-[15], compensation techniques [9], [16]-[20], or more recently BER analysis [21]. The estimation and/or compensation of IQ imbalances along with CFO have been studied in [17], [18], and [22]-[25]. However, pilot-data power allocation has not been addressed under the foregoing scenario. Under a fixed total power constraint, more pilot power reduces data detection quality, whereas less pilot power reduces the channel estimation quality. Hence, pilot-data power allocation poses a tradeoff, and it is of interest to find its solution in the presence of IQ imbalance and to know the effect CFO plays on the same. Toward this end, we formulate a lower bound on the ergodic capacity and optimize it to find the pilot-data power allocation and study how IQ imbalance and CFO affect the optimizing power allocation.

We consider two scenarios for our analysis, i.e., 1) the fast fading case, where the channel varies with every OFDM symbol, and 2) the slow fading case, where the channel is constant over a block of several OFDM symbols, and obtain the power allocation results.

Notations: Bold font denotes vector/matrix. Regular font denotes scalar. $(\cdot)^{T},(\cdot)^{*}$, and $(\cdot)^{H}$ denote transpose, complex conjugate, and conjugate transpose, respectively. $\boldsymbol{I}_{N}\left(\mathbf{0}_{N_{1} \times N_{2}}\right)$ denotes the identity (all zeros) matrix of size $N\left(N_{1} \times N_{2}\right)$. $\|\boldsymbol{x}\|$ denotes norm of vector $\boldsymbol{x} . \boldsymbol{A}(j, i)$ denotes the element at $j$ th row and $i$ th column of vector/matrix $\boldsymbol{A} .\{f(j, i)\}_{j \in J_{1}, i \in J_{2}}$ denotes the matrix with $f(j, i)$ in the $(j, i)$ th location; if no 
ranges are specified for $j, i$, then $j=1, \ldots, N_{R}$ and $i=$ $1, \ldots, N_{T}$; for matrix $\boldsymbol{f}_{j, i}$, which is a function of $j$ and $i$, $\left\{\boldsymbol{f}_{j, i}\right\}_{j \in J_{1}, i \in J_{2}}$ denotes a larger matrix with $\boldsymbol{f}_{j, i}$ in the $(j, i)$ th subblock location, where each subblock is of the same size as $\boldsymbol{f}_{j, i} . \boldsymbol{\Lambda}_{\boldsymbol{x}}$ denotes a diagonal matrix with elements of vector $\boldsymbol{x}$ on its diagonal. $\mathcal{C N}$ denotes complex Gaussian. i.i.d. denotes independent and identically distributed. $\operatorname{diag}(\boldsymbol{A})$ denotes a diagonal matrix with the diagonal elements of $\boldsymbol{A}$ on its diagonal. $\operatorname{nondiag}(\boldsymbol{A})$ denotes a matrix with zeros on the diagonal and the nondiagonal elements same as the corresponding elements in $A$.

\section{SYSTEM MODEL}

We consider a MIMO-OFDM system with $N_{T}$ transmit antennas, $N_{R}$ receive antennas, and $N$ subcarriers. The MIMO system may be spatially correlated, in which case we assume the widely used Kronecker correlation model with transmit and receive correlation matrices denoted by $\boldsymbol{R}_{T}$ and $\boldsymbol{R}_{R}$, respectively. The model also covers spatially uncorrelated channels where $\boldsymbol{R}_{T}$ and $\boldsymbol{R}_{R}$ are diagonal matrices. We do not assume either the transmitter or the receiver has knowledge of the spatial correlation. Let $L_{1}$ denote the maximum number of sample-spaced channel taps among all the transmit-receive antenna pairs.

As in practical systems, we assume that the carrier frequency offset (CFO) is compensated during frequency synchronization (e.g., based on preamble symbols). Thus, there will be a residual CFO, which is small, and we analyze its effect on the system performance.

In MIMO systems, different RF chains corresponding to different antennas may give rise to different IQ imbalances. Hence, the IQ parameters depend on the transmit and receive antenna indices. The IQ gains and phase offsets at the $i$ th transmit antenna are denoted by $\left\{a_{t, i}^{I}, a_{t, i}^{Q}\right\}$ and $\left\{\theta_{t, i}^{I}, \theta_{t, i}^{Q}\right\}$. The frequency-selective IQ imbalance introduced throughout baseband and RF chains (including D/A converters, amplifiers, and pulse shaping filters) is typically modeled by filters. Such filters for the I and $\mathrm{Q}$ branches of the $i$ th transmit antenna are denoted by $g_{t, i}^{I}(t)$ and $g_{t, i}^{Q}(t)$. The corresponding parameters at the $j$ th receive antenna are denoted by $a_{r, j}^{I}, a_{r, j}^{Q}, \theta_{r, j}^{I}, \theta_{r, j}^{Q}$, $g_{r, j}^{I}(t)$, and $g_{r, j}^{Q}(t)$. Referring to [13, Fig. 13], starting from the bandpass system that includes the effects of IQ parameters and the filters above, between any transmit-receive antenna pair, after deriving the equivalent low-pass system, we see that the overall system acts like a summation of two systems, one of which sees the original input as its input (which we call the direct system), and the other of which sees the conjugate of the input as its input (which we call the mirror system). If we denote the impulse responses of the direct and mirror systems at the $i$ th transmit antenna by $g_{T, i}^{D}(t), g_{T, i}^{M}(t)$ and those at the $j$ th receive antenna by $g_{R, j}^{D}(t), g_{R, j}^{M}(t)$, we observe that these are related to the IQ imbalance parameters by

$$
\begin{aligned}
& g_{T, i}^{D}(t)=\frac{1}{2}\left[a_{t, i}^{I} e^{j \theta_{t, i}^{I}} g_{t, i}^{I}(t)+a_{t, i}^{Q} e^{j \theta_{t, i}^{Q}} g_{t, i}^{Q}(t)\right] \\
& g_{T, i}^{M}(t)=\frac{1}{2}\left[a_{t, i}^{I} e^{j \theta_{t, i}^{I}} g_{t, i}^{I}(t)-a_{t, i}^{Q} e^{j \theta_{t, i}^{Q}} g_{t, i}^{Q}(t)\right]
\end{aligned}
$$

$$
\begin{aligned}
& g_{R, j}^{D}(t)=\frac{1}{2}\left[a_{r, j}^{I} e^{-j \theta_{r, j}^{I}} g_{r, j}^{I}(t)+a_{r, j}^{Q} e^{-j \theta_{r, j}^{Q}} g_{r, j}^{Q}(t)\right] \\
& g_{R, j}^{M}(t)=\frac{1}{2}\left[a_{r, j}^{I} e^{j \theta_{r, j}^{I}} g_{r, j}^{I}(t)-a_{r, j}^{Q} e^{j \theta_{r, j}^{Q}} g_{r, j}^{Q}(t)\right] .
\end{aligned}
$$

We denote the convolution matrices corresponding to the foregoing filters by $\boldsymbol{G}_{T, i}^{D}, \boldsymbol{G}_{T, i}^{M}, \boldsymbol{G}_{R, j}^{D}$, and $\boldsymbol{G}_{R, j}^{M}$, respectively. We denote the maximum number of taps in each of these filters by $Z$. Then, the dimensions of these matrices are $N_{1} \times N_{1}+Z-$ 1 , where $N_{1}$ will become clear from the context of their usage. We also normalize the channel taps in each of the IQ filters at the transmit and receive sides to have unit energy in each (i.e., the sum of energies in all taps in I and Q filters is unity at each transmit and receive antenna).

Let $\tilde{\boldsymbol{x}}_{i}$ denote the $\left(N+N_{\mathrm{CP}}\right) \times 1$ time-domain data signal at the $i$ th transmit antenna, where $N_{\mathrm{CP}}$ is the length of the cyclic prefix, and $\tilde{\boldsymbol{x}} \triangleq\left[\tilde{\boldsymbol{x}}_{1}^{T}, \ldots, \tilde{\boldsymbol{x}}_{N_{T}}^{T}\right]^{T}$. In the following, we will use the same notation for the pilot signals as that used for the data signals, but with the letter " $s / S$ " in place of " $x / X$ " as used for data symbols. Henceforth, we will use without defining terms related to pilot signals once we define similar terms for data signals. The effect of IQ imbalance at the transmitter and receiver sides is to create an additional conjugate signal in addition to the original signal. The output of the IQ filters at the $i$ th transmit antenna is, thus, given by

$$
\boldsymbol{y}_{1, i}=\boldsymbol{G}_{T, i}^{D}\left(\tilde{\boldsymbol{x}}_{i}+\tilde{\boldsymbol{s}}_{i}\right)+\boldsymbol{G}_{T, i}^{M}\left(\tilde{\boldsymbol{x}}_{i}^{*}+\tilde{\boldsymbol{s}}_{i}^{*}\right) .
$$

Here, $\boldsymbol{y}_{1, i}$ is of size $\left(N+N_{\mathrm{CP}}-Z+1\right) \times 1$. Then, we define $\boldsymbol{y}_{1} \triangleq\left[\boldsymbol{y}_{1,1}^{T}, \ldots, \boldsymbol{y}_{1, N_{T}}^{T}\right]^{T}$. After going through the channel and undergoing downconversion at the receiver, the signal at the $j$ th receive antenna, prior to the receive-side IQ imbalance, can be written as

$$
\boldsymbol{y}_{2, j}=\boldsymbol{W}(\nu)\left[\boldsymbol{H}_{j 1}, \ldots, \boldsymbol{H}_{j N_{T}}\right] \boldsymbol{y}_{1}+\boldsymbol{w}_{j}
$$

where $\boldsymbol{W}(\nu)$ is the diagonal CFO matrix with entries $e^{(-2 \pi j k \nu / N)}, k=0,1, \ldots, N+Z-1$, on the diagonal, $\nu$ is the CFO normalized by the OFDM subcarrier spacing, $\boldsymbol{H}_{j i}$ is the convolution matrix corresponding to $\boldsymbol{h}_{j i} \triangleq$ $\left[h_{j i}^{(0)}, h_{j i}^{(1)}, \ldots, h_{j i}^{\left(L_{1}-1\right)}\right]^{T}, h_{j i}^{(l)}$ is the $l$ th tap channel gain between the $i$ th transmit and $j$ th receive antenna, and $\boldsymbol{w}_{j}$ is the zero mean circularly symmetric complex Gaussian (ZMCSCG) noise vector at the $j$ th receive antenna, having covariance matrix $\sigma_{w}^{2} \boldsymbol{I}_{N+Z-1}$. After going through the receive-side IQ filters, the signal at the $j$ th receive antenna can be written as

$$
\begin{aligned}
\boldsymbol{r}_{j}= & \boldsymbol{G}_{R, j}^{D} \boldsymbol{y}_{2, j}+\boldsymbol{G}_{R, j}^{M} \boldsymbol{y}_{2, j}^{*} \\
= & {\left[\overline{\boldsymbol{A}}_{1, j 1} \boldsymbol{H}_{j 1}, \ldots, \overline{\boldsymbol{A}}_{1, j N_{T}} \boldsymbol{H}_{j N_{T}}\right](\tilde{\boldsymbol{x}}+\tilde{\boldsymbol{s}}) } \\
& +\left[\overline{\boldsymbol{A}}_{2, j 1} \boldsymbol{H}_{j 1^{*}}, \ldots, \overline{\boldsymbol{A}}_{2, j N_{T}} \boldsymbol{H}_{j N_{T}}^{*}\right](\tilde{\boldsymbol{x}}+\tilde{\boldsymbol{s}}) \\
& +\left[\overline{\boldsymbol{B}}_{1, j 1} \boldsymbol{H}_{j 1}, \ldots, \overline{\boldsymbol{B}}_{1, j N_{T}} \boldsymbol{H}_{j N_{T}}\right]\left(\tilde{\boldsymbol{x}}^{*}+\tilde{\boldsymbol{s}}^{*}\right) \\
& +\left[\overline{\boldsymbol{B}}_{2, j 1} \boldsymbol{H}_{j 1^{*}}, \ldots, \overline{\boldsymbol{B}}_{2, j N_{T}} \boldsymbol{H}_{j N_{T}}^{*}\right]\left(\tilde{\boldsymbol{x}}^{*}+\tilde{\boldsymbol{s}}^{*}\right)+\boldsymbol{n}_{j}
\end{aligned}
$$

where

$$
\begin{aligned}
\overline{\boldsymbol{A}}_{1, j i} \triangleq \boldsymbol{G}_{R, j}^{D} \boldsymbol{W}(\nu) \boldsymbol{G}_{T, i}^{D}, \overline{\boldsymbol{A}}_{2, j i} \triangleq \boldsymbol{G}_{R, j}^{M} \boldsymbol{W}(\nu)^{*} \boldsymbol{G}_{T, i}^{M *} \\
\overline{\boldsymbol{B}}_{1, j i} \triangleq \boldsymbol{G}_{R, j}^{D} \boldsymbol{W}(\nu) \boldsymbol{G}_{T, i}^{M}, \overline{\boldsymbol{B}}_{2, j i} \triangleq \boldsymbol{G}_{R, j}^{M} \boldsymbol{W}^{*}(\nu) \boldsymbol{G}_{T, i}^{D *}
\end{aligned}
$$


and we have used the commutativity property of convolution to interchange the channel and IQ filter matrices. The noise $\boldsymbol{n}_{j} \triangleq$ $\boldsymbol{G}_{R, j}^{D} \boldsymbol{w}_{j}+\boldsymbol{G}_{R, j}^{M} \boldsymbol{w}_{j}^{*}$ is zero-mean complex Gaussian with covariance and pseudocovariance given by

$$
\begin{gathered}
\boldsymbol{R}_{n_{j}} \triangleq \mathrm{E}\left[\boldsymbol{n}_{j} \boldsymbol{n}_{j}^{H}\right]=\sigma_{w}^{2}\left(\boldsymbol{G}_{R, j}^{D} \boldsymbol{G}_{R, j}^{D H}+\boldsymbol{G}_{R, j}^{M} \boldsymbol{G}_{R, j}^{M H}\right) \\
\boldsymbol{M}_{n_{j}} \triangleq \mathrm{E}\left[\boldsymbol{n}_{j} \boldsymbol{n}_{j}^{T}\right]=\sigma_{w}^{2}\left(\boldsymbol{G}_{R, j}^{D} \boldsymbol{G}_{R, j}^{M T}+\boldsymbol{G}_{R, j}^{M} \boldsymbol{G}_{R, j}^{D T}\right) .
\end{gathered}
$$

In the following work, we consider the IQ imbalances at the receive side are only mildly frequency selective or frequency flat. In this case, from the normalization of $\mathrm{I}$ and $\mathrm{Q}$ filter tap energies, we can approximate $\boldsymbol{R}_{\boldsymbol{n}_{j}} \approx \sigma_{w}^{2} \boldsymbol{I}_{N_{R}} \triangleq \sigma_{n}^{2} \boldsymbol{I}_{N_{R}}$. The pseudocovariance of $\boldsymbol{n}_{j}$ can be simplified as $\boldsymbol{M}_{n_{j}}=$ $\left(\sigma_{w}^{2} / 2\right)\left[\left(a_{r, j}^{I}\right)^{2} \cos \left(2 \theta_{r, j}^{I}\right)-\left(a_{r, j}^{Q}\right)^{2} \cos \left(2 \theta_{r, j}^{Q}\right)\right] \boldsymbol{I}_{N_{R}}$. We have $(1 / 2)\left[\left(a_{r, j}^{I}\right)^{2} \cos \left(2 \theta_{r, j}^{I}\right)-\left(a_{r, j}^{Q}\right)^{2} \cos \left(2 \theta_{r, j}^{Q}\right)\right] \ll 1$, and thus, the pseudocovariance is approximated as

$$
\boldsymbol{M}_{\boldsymbol{n}_{j}} \approx \mathbf{0}, \forall j \in\left\{1,2, \ldots, N_{R}\right\} .
$$

To facilitate further analysis, we define the following terms:

$$
\begin{aligned}
\overline{\boldsymbol{A}}_{1, j} \triangleq\left[\overline{\boldsymbol{A}}_{1, j 1}, \ldots, \overline{\boldsymbol{A}}_{1, j N_{T}}\right], \overline{\boldsymbol{A}}_{2, j} \triangleq\left[\overline{\boldsymbol{A}}_{2, j 1}, \ldots, \overline{\boldsymbol{A}}_{2, j N_{T}}\right] \\
\overline{\boldsymbol{B}}_{1, j} \triangleq\left[\overline{\boldsymbol{B}}_{1, j 1}, \ldots, \overline{\boldsymbol{B}}_{1, j N_{T}}\right], \overline{\boldsymbol{B}}_{2, j} \triangleq\left[\overline{\boldsymbol{B}}_{2, j 1}, \ldots, \overline{\boldsymbol{B}}_{2, j N_{T}}\right] \\
\overline{\boldsymbol{A}}_{1} \triangleq\left\{\overline{\boldsymbol{A}}_{1, j i}\right\}_{j, i}, \overline{\boldsymbol{A}}_{2} \triangleq\left\{\overline{\boldsymbol{A}}_{2, j i}\right\}_{j, i}, \overline{\boldsymbol{B}}_{1} \triangleq\left\{\overline{\boldsymbol{B}}_{1, j i}\right\}_{j, i} \\
\overline{\boldsymbol{B}}_{2} \triangleq\left\{\overline{\boldsymbol{B}}_{2, j i}\right\}_{j, i}, i=1, \ldots, N_{T} ; j=1, \ldots, N_{R} .
\end{aligned}
$$

Then, stacking the vectors from all the receive antennas, we can write as

$$
\begin{aligned}
\boldsymbol{r}= & {\left[\left\{\overline{\boldsymbol{A}}_{1, j i} \boldsymbol{H}_{j i}\right\}_{j, i}+\left\{\overline{\boldsymbol{A}}_{2, j i} \boldsymbol{H}_{j i}^{*}\right\}_{j, i}\right](\tilde{\boldsymbol{x}}+\tilde{\boldsymbol{s}}) } \\
& +\left[\left\{\overline{\boldsymbol{B}}_{1, j i} \boldsymbol{H}_{j i}\right\}_{j, i}+\left\{\overline{\boldsymbol{B}}_{2, j i} \boldsymbol{H}_{j i}^{*}\right\}_{j, i}\right]\left(\tilde{\boldsymbol{x}}^{*}+\tilde{\boldsymbol{s}}^{*}\right)+\boldsymbol{n}
\end{aligned}
$$

where $\boldsymbol{n}=\left[\boldsymbol{n}_{1}^{T}, \ldots, \boldsymbol{n}_{N_{R}}^{T}\right]^{T}$. Let $\boldsymbol{F}$ be the $N \times N$ standard discrete Fourier transform (DFT) matrix, $\boldsymbol{f}_{k}$ be the $k$ th row of $\boldsymbol{F}, \boldsymbol{F}_{L}$ be the matrix with the first $L$ columns of $\boldsymbol{F}$, and $\boldsymbol{F}_{L, k}$ be the $k$ th row of $\boldsymbol{F}_{L}$.

Transforming to frequency domain and defining $\boldsymbol{R} \triangleq$ $\left\{\boldsymbol{R}_{j}\right\}_{j, 1}$ with $\boldsymbol{R}_{j} \triangleq \boldsymbol{F r}_{j}$, we have

$$
\begin{aligned}
\boldsymbol{R}= & \frac{1}{N}\left[\left\{\boldsymbol{F} \overline{\boldsymbol{A}}_{1, j i} \boldsymbol{H}_{j i} \tilde{\boldsymbol{P}} \boldsymbol{F}^{H}+\boldsymbol{F} \overline{\boldsymbol{A}}_{2, j i} \boldsymbol{H}_{j i} \tilde{\boldsymbol{P}} \boldsymbol{F}^{H}\right\}_{j, i}\right] \times(\boldsymbol{X}+\boldsymbol{S}) \\
& +\frac{1}{N}\left[\left\{\boldsymbol{F} \overline{\boldsymbol{B}}_{1, j i} \boldsymbol{H}_{j i} \tilde{\boldsymbol{P}} \boldsymbol{F}^{H}+\boldsymbol{F} \overline{\boldsymbol{B}}_{2, j i} \boldsymbol{H}_{j i} \tilde{\boldsymbol{P}} \boldsymbol{F}^{H}\right\}_{j, i}\right] \\
& \times\left(\boldsymbol{X}_{-}^{*}+\boldsymbol{S}_{-}^{*}\right)+\boldsymbol{N}_{k}
\end{aligned}
$$

where $\boldsymbol{X} \triangleq\left[\boldsymbol{X}_{1}^{T}, \ldots, \boldsymbol{X}_{N_{T}}^{T}\right]^{T}, \boldsymbol{X}_{i} \triangleq\left[X_{0, i}, X_{1, i} \ldots, X_{(N-1), i}\right]^{T}$, $\boldsymbol{X}_{-} \triangleq\left[\boldsymbol{X}_{-, 1}^{T}, \ldots, \boldsymbol{X}_{-, N_{T}}^{T}\right]^{T}, \quad \boldsymbol{X}_{-, i} \triangleq\left[X_{-0, i}, X_{-1, i} \ldots\right.$, $\left.X_{-(N-1), i}\right]^{T}$, and $X_{k, i}$ and $X_{-k, i}$ are the $k$ th and $(N-k)$ th tone data symbols, respectively, at the $i$ th transmit antenna. In deriving the above, we have used $\tilde{\boldsymbol{x}}=\left\{\tilde{\boldsymbol{P}} \boldsymbol{x}_{i}\right\}_{i, 1}$ for $\boldsymbol{x}_{i}=(1 / N) \boldsymbol{F}^{H} \boldsymbol{X}_{i}$, where $\tilde{\boldsymbol{P}} \triangleq\left[\begin{array}{l|l|l}\mathbf{0} & \boldsymbol{I}_{N_{\mathrm{CP}}} \\ \hline & \boldsymbol{I}_{N}\end{array}\right]$ is the permutation matrix, $\boldsymbol{x}_{i}$ is the $N \times 1$ transmit signal (i.e., without the cyclic prefix), and $\boldsymbol{x} \triangleq\left[\boldsymbol{x}_{1}^{T}, \ldots, \boldsymbol{x}_{N_{T}}^{T}\right]^{T}$. We note that in the absence of residual CFO (i.e., for identity matrix $\boldsymbol{W}(\nu)$ ), each of the matrices

$$
\begin{aligned}
& \boldsymbol{A}_{1, j i} \triangleq \boldsymbol{F} \overline{\boldsymbol{A}}_{1, j i} \boldsymbol{H}_{j i} \tilde{\boldsymbol{P}} \boldsymbol{F}^{H}, \boldsymbol{A}_{2, j i} \triangleq \boldsymbol{F} \overline{\boldsymbol{A}}_{2, j i} \boldsymbol{H}_{j i} \tilde{\boldsymbol{P}} \boldsymbol{F}^{H} \\
& \boldsymbol{B}_{1, j i} \triangleq \boldsymbol{F} \overline{\boldsymbol{B}}_{1, j i} \boldsymbol{H}_{j i} \tilde{\boldsymbol{P}} \boldsymbol{F}^{H} \text { and } \boldsymbol{B}_{2, j i} \triangleq \boldsymbol{F} \overline{\boldsymbol{B}}_{2, j i} \boldsymbol{H}_{j i} \tilde{\boldsymbol{P}} \boldsymbol{F}^{H}
\end{aligned}
$$

would be diagonal. However, for nonidentity $\boldsymbol{W}(\nu)$, these matrices are nondiagonal, resulting in intercarrier interference. In addition, due to the conjugation effect produced by the IQ imbalance, there is interference from the mirror tone for any given tone.

We separate the desired signal and interference terms in the foregoing expression by rewriting as

$$
\boldsymbol{R}=\boldsymbol{P}(\boldsymbol{X}+\boldsymbol{S})+\boldsymbol{Q}\left(\boldsymbol{X}_{-}^{*}+\boldsymbol{S}_{-}^{*}\right)+\boldsymbol{V}+\boldsymbol{N}
$$

where

$$
\begin{aligned}
\boldsymbol{P} \triangleq & {\left[\left\{\operatorname{diag}\left(\boldsymbol{A}_{1, j i}\right)+\operatorname{diag}\left(\boldsymbol{A}_{2, j i}\right)\right\}_{j, i}\right] } \\
\boldsymbol{Q} \triangleq & {\left[\left\{\operatorname{diag}\left(\boldsymbol{B}_{1, j i, k}\right)+\operatorname{diag}\left(\boldsymbol{B}_{2, j i}\right)\right\}_{j, i}\right] } \\
\boldsymbol{V} \triangleq & {\left[\left\{\boldsymbol{A}_{1, j i}+\boldsymbol{A}_{2, j i}-\operatorname{diag}\left(\boldsymbol{A}_{1, j i}\right)-\operatorname{diag}\left(\boldsymbol{A}_{2, j i}\right)\right\}_{j, i}\right] } \\
& \times(\boldsymbol{X}+\boldsymbol{S}) \\
& +\left[\left\{\boldsymbol{B}_{1, j i}+\boldsymbol{B}_{2, j i}-\operatorname{diag}\left(\boldsymbol{B}_{1, j i}\right)-\operatorname{diag}\left(\boldsymbol{B}_{2, j i}\right)\right\}_{j, i}\right] \\
& \times\left(\boldsymbol{X}_{-}^{*}+\boldsymbol{S}_{-}^{*}\right) .
\end{aligned}
$$

For later usage, we will also define

$$
\begin{aligned}
\boldsymbol{V}_{j_{0}} \triangleq & {\left[\left\{\operatorname{nondiag}\left(\boldsymbol{A}_{1, j i}\right)+\operatorname{nondiag}\left(\boldsymbol{A}_{2, j i}\right)\right\}_{j=j_{0}, i=1, \ldots N_{T}}\right] } \\
& \times(\boldsymbol{X}+\boldsymbol{S}) \\
& +\left[\left\{\operatorname{nondiag}\left(\boldsymbol{B}_{1, j i}\right)+\operatorname{nondiag}\left(\boldsymbol{B}_{2, j i}\right)\right\}_{j=j_{0}, i=1, \ldots N_{T}}\right] \\
& \times\left(\boldsymbol{X}_{-}^{*}+\boldsymbol{S}_{-}^{*}\right)
\end{aligned}
$$

as the frequency domain interference at the $j_{0}$ th receive antenna, $V_{k, j}$ as the interference on the $k$ th tone at the $j$ th receive antenna, and $\overline{\boldsymbol{V}}_{k} \triangleq\left[V_{k, 1}, V_{k, 2}, \ldots, V_{k, N_{R}}\right]^{T}$ as the $k$ th tone interference at all receive antennas.

To see the direct and mirror channels due to IQ imbalance and the interference terms due to $\mathrm{CFO}$ in the time domain, we convert back the above into time domain to obtain

$$
\boldsymbol{r}=\left\{\boldsymbol{P}_{\mathrm{circ}, j i}\right\}_{j, i}(\boldsymbol{x}+\boldsymbol{s})+\left\{\boldsymbol{Q}_{\mathrm{circ}, j i}\right\}_{j, i}\left(\boldsymbol{x}^{*}+\boldsymbol{s}^{*}\right)+\boldsymbol{v}+\boldsymbol{n}
$$

where

$$
\begin{aligned}
\left\{\boldsymbol{P}_{\text {circ }, j i}\right\}_{j, i} & \triangleq \\
\triangleq & \frac{1}{N}\left\{\boldsymbol{F}^{H} \operatorname{diag}\left(\boldsymbol{A}_{1, j i}\right) \boldsymbol{F}+\boldsymbol{F}^{H} \operatorname{diag}\left(\boldsymbol{A}_{2, j i}\right) \boldsymbol{F}\right\}_{j, i} \\
\left\{\boldsymbol{Q}_{\mathrm{circ}, j i}\right\}_{j, i} & \triangleq \frac{1}{N}\left\{\boldsymbol{F}^{H} \operatorname{diag}\left(\boldsymbol{B}_{1, j i, k}\right) \boldsymbol{F}+\boldsymbol{F}^{H} \operatorname{diag}\left(\boldsymbol{B}_{2, j i}\right) \boldsymbol{F}\right\}_{j, i} \\
\boldsymbol{v} \triangleq & \frac{1}{N}\left[\left\{\boldsymbol{F}^{H}\left(\operatorname{nondiag}\left(\boldsymbol{A}_{1, j i}\right)+\operatorname{nondiag}\left(\boldsymbol{A}_{2, j i}\right)\right) \boldsymbol{F}\right\}_{j, i}\right] \\
& \times(\boldsymbol{x}+\boldsymbol{s}) \\
& +\frac{1}{N}\left[\left\{\boldsymbol{F}^{H}\left(\operatorname{nondiag}\left(\boldsymbol{B}_{1, j i}\right)+\operatorname{nondiag}\left(\boldsymbol{B}_{2, j i}\right)\right) \boldsymbol{F}\right\}_{j, i}\right] \\
& \times\left(\boldsymbol{x}^{*}+\boldsymbol{s}^{*}\right)
\end{aligned}
$$




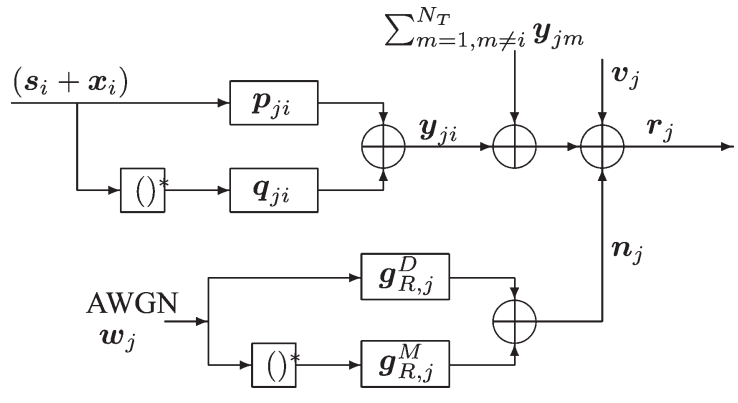

Fig. 1. Signal model for a MIMO-OFDM system with frequency-selective IQ imbalance and residual CFO, where $\boldsymbol{g}_{R, j}^{D}$ and $\boldsymbol{g}_{R, j}^{M}$ are discrete versions of filters $g_{R, j}^{D}(t)$ and $g_{R, j}^{M}(t)$, respectively. The direct and mirror channels $\boldsymbol{p}_{j i}$ and $\boldsymbol{q}_{j i}$ include the effects of IQ imbalance and CFO, whereas $\boldsymbol{v}_{j}$ is the CFOinduced intercarrier interference.

and $\boldsymbol{P}_{\text {circ }, j i}$ and $\boldsymbol{Q}_{\mathrm{circ}, j i}$ are circulant matrices from the definition above. The overall system model with IQ imbalance and CFO is shown in Fig. 1.

\section{Channel Estimation}

To design the pilots for channel estimation, we need to know the maximum number of taps of the equivalent time domain channel incorporating IQ imbalance and the underlying channel. If the maximum of the number of taps between all the transmit-receive antenna pairs in the underlying channel is $L_{1}$, then the maximum number of channel taps in each of the effective direct and mirror channels whose circulant convolution matrix is given by $\boldsymbol{P}_{\text {circ }, j i}$ and $\boldsymbol{Q}_{\text {circ }, j i}$, respectively, is $L \triangleq L_{1}+2 Z-2$. (Proof is given in the Appendix.)

Thus, the effective direct and mirror channels have $L$ taps each. For channel estimation purposes, we may write (22) as

$$
\boldsymbol{r}=\boldsymbol{I}_{N_{R}} \otimes\left[\boldsymbol{X}_{\mathrm{circ}}+\boldsymbol{S}_{\mathrm{circ}}\right] \boldsymbol{p}+\boldsymbol{I}_{N_{R}} \otimes\left[\boldsymbol{X}_{\mathrm{circ}}^{*}+\boldsymbol{S}_{\mathrm{circ}}^{*}\right] \boldsymbol{q}+\boldsymbol{v}+\boldsymbol{n}
$$

where $\boldsymbol{p}=\left[\boldsymbol{p}_{1}^{T}, \ldots, \boldsymbol{p}_{N_{T}}^{T}\right]^{T}, \boldsymbol{q}=\left[\boldsymbol{q}_{1}^{T}, \ldots, \boldsymbol{q}_{N_{T}}^{T}\right]^{T}, \boldsymbol{p}_{j}=\left[\boldsymbol{p}_{j 1}^{T}\right.$, $\left.\ldots, \boldsymbol{p}_{j N_{T}}^{T}\right]^{T}, \boldsymbol{q}_{j}=\left[\boldsymbol{q}_{j 1}^{T}, \ldots, \boldsymbol{q}_{j N_{T}}^{T}\right]^{T}, \boldsymbol{p}_{j i}=\left[p_{j i}^{(0)}, \ldots, p_{j i}^{(L-1)}\right]^{T}$, $\boldsymbol{q}_{j i}=\left[q_{j i}^{(0)}, \ldots, q_{j i}^{(L-1)}\right]^{T}$, and $p_{j i}^{(l)}$ and $q_{j i}^{(l)}, l=0,1, \ldots, L-$ 1 , are the $l$ th tap channel gains for the effective direct and mirror channels. $\boldsymbol{X}_{\text {circ }} \triangleq\left[\boldsymbol{X}_{\text {circ, } 1}, \ldots, \boldsymbol{X}_{\text {circ, } N_{T}}\right]$, with $\boldsymbol{X}_{\text {circ }, i} \triangleq$ $(1 / N) \boldsymbol{F}^{H} \boldsymbol{\Lambda}_{\boldsymbol{X}_{i}} \boldsymbol{F}$ being the circulant convolution matrices corresponding to $\boldsymbol{x}_{i}$. We note here that $\boldsymbol{P}_{\text {circ }, j i}$ and $\boldsymbol{Q}_{\text {circ }, j i}$ are related to $\boldsymbol{p}_{j i}$ and $\boldsymbol{q}_{j i}$ as $\boldsymbol{P}_{\text {circ }, j i}=(1 / N) \boldsymbol{F}^{H} \boldsymbol{\Lambda}_{\boldsymbol{F} p_{j i}} \boldsymbol{F}$ and $\boldsymbol{Q}_{\text {circ }, j i}=(1 / N) \boldsymbol{F}^{H} \boldsymbol{\Lambda}_{\boldsymbol{F} q_{j i}} \boldsymbol{F}$, respectively.

In practical systems, the statistics of channel and device imperfections are unknown, and they might be nonstationary as well. As a result, we choose least-square (LS) channel estimators. These estimators are also maximum likelihood with the linear Gaussian signal model, which are known to achieve the Cramer-Rao bound. The LS estimates of channels $p$ and $q$ are

$$
\begin{gathered}
\hat{\boldsymbol{p}}=\left(\left[\boldsymbol{I}_{N_{R}} \otimes \boldsymbol{S}_{\mathrm{circ}}\right]^{H}\left[\boldsymbol{I}_{N_{R}} \otimes \boldsymbol{S}_{\mathrm{circ}}\right]\right)^{-1}\left[\boldsymbol{I}_{N_{R}} \otimes \boldsymbol{S}_{\mathrm{circ}}\right]^{H} \boldsymbol{r} \\
\hat{\boldsymbol{q}}=\left(\left[\boldsymbol{I}_{N_{R}} \otimes \boldsymbol{S}_{\mathrm{circ}}\right]^{T}\left[\boldsymbol{I}_{N_{R}} \otimes \boldsymbol{S}_{\mathrm{circ}}^{*}\right]\right)^{-1}\left[\boldsymbol{I}_{N_{R}} \otimes \boldsymbol{S}_{\mathrm{circ}}\right]^{T} \boldsymbol{r} \\
\hat{\boldsymbol{p}}_{j}=\left(\boldsymbol{S}_{\mathrm{circ}}^{H} \boldsymbol{S}_{\mathrm{circ}}\right)^{-1} \boldsymbol{S}_{\mathrm{circ}}^{H} \boldsymbol{r}_{j} \triangleq\left[\hat{\boldsymbol{p}}_{j 1}^{T}, \hat{\boldsymbol{p}}_{j 2}^{T}, \ldots, \hat{\boldsymbol{p}}_{j N_{T}}^{T}\right]^{T} \\
\hat{\boldsymbol{q}}_{j}=\left(\boldsymbol{S}_{\mathrm{circ}}^{T} \boldsymbol{S}_{\mathrm{circ}}^{*}\right)^{-1} \boldsymbol{S}_{\mathrm{circ}}^{T} \boldsymbol{r}_{j} \triangleq\left[\hat{\boldsymbol{q}}_{j 1}^{T}, \hat{\boldsymbol{q}}_{j 2}^{T}, \ldots, \hat{\boldsymbol{q}}_{j N_{T}}^{T}\right]^{T}
\end{gathered}
$$

where $\hat{\boldsymbol{p}}_{j i}$ and $\hat{\boldsymbol{q}}_{j i}$ are the estimates of $\boldsymbol{p}_{j i}$ and $\boldsymbol{q}_{j i}$, respectively. From the foregoing equations, we see that the channels can be estimated at each receive antenna independently as well. Let $\Delta \boldsymbol{p}, \Delta \boldsymbol{q}, \Delta \boldsymbol{p}_{j}$, and $\Delta \boldsymbol{q}_{j}$ denote the corresponding estimation errors. Then

$$
\begin{aligned}
& \Delta \boldsymbol{p}_{j}=\left(\boldsymbol{S}_{\text {circ }}^{H} \boldsymbol{S}_{\text {circ }}\right)^{-1} \boldsymbol{S}_{\text {circ }}^{H}\left(\boldsymbol{n}_{j}+\boldsymbol{v}_{j}\right) \\
& \Delta \boldsymbol{q}_{j}=\left(\boldsymbol{S}_{\text {circ }}^{T} \boldsymbol{S}_{\text {circ }}^{*}\right)^{-1} \boldsymbol{S}_{\text {circ }}^{T}\left(\boldsymbol{n}_{j}+\boldsymbol{v}_{j}\right)
\end{aligned}
$$

where $\boldsymbol{v}_{j}$ is obtained by fixing $j$ and varying only $i$ in (25). The error covariances and MSEs are given as

$$
\begin{aligned}
\boldsymbol{R}_{\Delta \boldsymbol{p}_{j}} & \triangleq \mathrm{E}\left[\Delta \boldsymbol{p}_{j} \Delta \boldsymbol{p}_{j}^{H}\right] \\
& =\left(\boldsymbol{S}_{\text {circ }}^{H} \boldsymbol{S}_{\text {circ }}\right)^{-1} \boldsymbol{S}_{\text {circ }}^{H}\left(\boldsymbol{R}_{\boldsymbol{n}_{j}}+\boldsymbol{R}_{\boldsymbol{v}_{j}}\right) \boldsymbol{S}_{\text {circ }}\left(\boldsymbol{S}_{\text {circ }}^{H} \boldsymbol{S}_{\text {circ }}\right)^{-1} \\
\boldsymbol{R}_{\Delta \boldsymbol{q}_{j}} & \triangleq \mathrm{E}\left[\Delta \boldsymbol{q}_{j} \Delta \boldsymbol{q}_{j}^{H}\right] \\
& =\left(\boldsymbol{S}_{\text {circ }}^{T} \boldsymbol{S}_{\text {circ }}^{*}\right)^{-1} \boldsymbol{S}_{\text {circ }}^{T}\left(\boldsymbol{R}_{\boldsymbol{n}_{j}}+\boldsymbol{R}_{\boldsymbol{v}_{j}}\right) \boldsymbol{S}_{\text {circ }}^{*}\left(\boldsymbol{S}_{\text {circ }}^{T} \boldsymbol{S}_{\text {circ }}^{*}\right)^{-1} \\
\operatorname{MSE}_{\boldsymbol{p}_{j}} & \triangleq \sigma_{\Delta p_{j}}^{2}=\operatorname{trace}\left[\boldsymbol{R}_{\left.\Delta \boldsymbol{p}_{j}\right]}\right. \\
\mathrm{MSE}_{\boldsymbol{q}_{j}} & \triangleq \sigma_{\Delta q_{j}}^{2}=\operatorname{trace}\left[\boldsymbol{R}_{\Delta \boldsymbol{q}_{j}}\right]=\operatorname{MSE}_{\boldsymbol{p}_{j}}
\end{aligned}
$$

where $\boldsymbol{R}_{\boldsymbol{v}_{j}} \triangleq \mathrm{E}\left[\boldsymbol{v}_{j} \boldsymbol{v}_{j}^{H}\right]$. We note that this method of estimating the direct and mirror channels is mainly efficient when the IQ imbalances are frequency selective, since in this case it becomes very difficult to separately estimate the IQ parameters and the channel [11], [13].

The residual CFO (after frequency synchronization) is typically very small, but the transmitter does not know its numerical value. Hence, under no CFO assumption, we select those designs that are known to minimize the estimation MSE and are optimal under the considered framework of estimating the combined response due to the channel and IQ parameters. In later sections, we will study the effect of CFO on the system performance. From [13], we know that the pilot designs that minimize the estimation MSE satisfy the following conditions:

$$
\begin{aligned}
\boldsymbol{S}_{\mathrm{circ}}^{H} \boldsymbol{S}_{\mathrm{circ}} & =P_{s} \boldsymbol{I}_{L N_{T}}, \boldsymbol{S}_{\mathrm{circ}}^{H} \boldsymbol{S}_{\mathrm{circ}}^{*}=\mathbf{0}_{L N_{T}} \\
\boldsymbol{S}_{\mathrm{circ}}^{H} \boldsymbol{X}_{\mathrm{circ}} & =\mathbf{0}_{L N_{T}}, \boldsymbol{S}_{\mathrm{circ}}^{H} \boldsymbol{X}_{\mathrm{circ}}^{*}=\mathbf{0}_{L N_{T}}
\end{aligned}
$$

where $P_{s}=\left\|s_{i}\right\|^{2}, \forall i$, is the total power of $N$ time-domain training samples at each transmit antenna. While these conditions are met by several pilot designs as laid out in [13], we will use one such design for illustration purposes, which is referred 
to as frequency-division multiplexing in [13]. In this design, pilots at different antennas are decoupled using orthogonal tones, and the mirror tone interferences are suppressed using mirror null tones. Such a design enables the estimation of direct and mirror channels at all the antennas. Further details and examples are described in [13]. Using the foregoing design, we get

$$
\begin{aligned}
& \boldsymbol{R}_{\Delta \boldsymbol{p}_{j}}=\frac{1}{P_{s}^{2}} \boldsymbol{S}_{\mathrm{circ}}^{H}\left(\boldsymbol{R}_{\boldsymbol{n}_{j}}+\boldsymbol{R}_{\boldsymbol{v}_{j}}\right) \boldsymbol{S}_{\mathrm{circ}} \\
& \boldsymbol{R}_{\Delta \boldsymbol{q}_{j}}=\frac{1}{P_{s}^{2}} \boldsymbol{S}_{\mathrm{circ}}^{T}\left(\boldsymbol{R}_{\boldsymbol{n}_{j}}+\boldsymbol{R}_{\boldsymbol{v}_{j}}\right) \boldsymbol{S}_{\mathrm{circ}}^{*} .
\end{aligned}
$$

\section{Channel Capacity Analysis}

In this section, we first find the ergodic channel capacity of a MIMO-OFDM system with IQ imbalance. Here, we consider the fast fading case wherein the channel varies with every OFDM symbol and is assumed to remain static within each symbol. The slow fading case will be dealt with in Section VI. Let the total transmit power at each transmit antenna be $P=$ $P_{x}+P_{s}$, where $P_{x}=\mathrm{E}\left\{\left\|\boldsymbol{x}_{i}\right\|^{2}\right\} \forall i$ is the total power in $N$ time-domain data samples at each transmit antenna. We also define $P_{X} \triangleq \mathrm{E}\left[\left|X_{k, i}\right|^{2}\right]$ as the average energy in each data tone symbol.

To find the capacity, we need to find the tone-wise frequencydomain expression for our system. Starting with (14), taking the DFT and writing the received vector on the $k$ th and $(N-k)$ th tones together, we obtain

$$
\begin{aligned}
\underbrace{\left[\begin{array}{c}
\overline{\boldsymbol{R}}_{k} \\
\overline{\boldsymbol{R}}_{-k}^{*}
\end{array}\right]}_{\tilde{\boldsymbol{r}}_{k}}=\underbrace{\left[\begin{array}{cc}
\boldsymbol{P}_{k} & \boldsymbol{Q}_{k} \\
\boldsymbol{Q}_{-k}^{*} & \boldsymbol{P}_{-k}^{*}
\end{array}\right]}_{\tilde{\boldsymbol{H}}_{k}} \underbrace{\left[\begin{array}{c}
\overline{\boldsymbol{X}}_{k} \\
\overline{\boldsymbol{X}}_{-k}^{*}
\end{array}\right]}_{\tilde{\boldsymbol{X}}_{k}}+\underbrace{\left[\begin{array}{c}
\overline{\boldsymbol{V}}_{k} \\
\overline{\boldsymbol{V}}_{-k}^{*}
\end{array}\right]}_{\tilde{\boldsymbol{V}}_{k}} \\
+\underbrace{\left[\begin{array}{c}
\boldsymbol{N}_{k} \\
\boldsymbol{N}_{-k}^{*}
\end{array}\right]}_{\tilde{\boldsymbol{N}}_{k}}, k \in \mathcal{I}^{+}
\end{aligned}
$$

where $\overline{\boldsymbol{R}}_{k} \triangleq\left\{\boldsymbol{f}_{k} \boldsymbol{r}_{j}\right\}_{j, 1}=\left[R_{k, 1}, \ldots, R_{k, N_{R}}\right]^{T}, R_{k, j}$ is the $k$ th tone signal at the $j$ th receive antenna, $\overline{\boldsymbol{X}}_{k} \triangleq\left\{\boldsymbol{f}_{k} \boldsymbol{x}_{i}\right\}_{i, 1}=$ $\left[X_{k, 1}, \ldots, X_{k, N_{T}}\right]^{T}$ is the $k$ th tone data vector, $\boldsymbol{P}_{k}=$ $\left\{\boldsymbol{f}_{k} \boldsymbol{p}_{j i}\right\}_{j, i}, \boldsymbol{Q}_{k}=\left\{\boldsymbol{f}_{k} \boldsymbol{q}_{j i}\right\}_{j, i}$, and $\mathcal{I}^{+}$is the set of data tones between 1 and $(N / 2-1)$. We note that due to the pilot design, for $k \in \mathcal{I}^{+}, \overline{\boldsymbol{S}}_{k}=\overline{\boldsymbol{S}}_{-k}^{*}=\mathbf{0}_{N_{T} \times 1}$, i.e., the data and pilot tones are kept orthogonal.

Stacking $\tilde{\boldsymbol{r}}_{k}$ for $k \in \mathcal{I}^{+}$, we get

$$
\begin{array}{r}
\underbrace{\left[\begin{array}{c}
\tilde{\boldsymbol{r}}_{k_{1}} \\
\vdots \\
\tilde{\boldsymbol{r}}_{k_{|\mathcal{I}+|}}
\end{array}\right]}_{\tilde{\boldsymbol{r}}}=\underbrace{\left[\begin{array}{ccc}
\tilde{\boldsymbol{H}}_{k_{1}} & \mathbf{0} & \ldots \\
\mathbf{0} & \ddots & \\
\vdots & & \tilde{\boldsymbol{H}}_{k_{|\mathcal{I}+|}}
\end{array}\right]}_{\tilde{\boldsymbol{H}}} \underbrace{\left[\begin{array}{c}
\tilde{\boldsymbol{X}}_{k_{1}} \\
\vdots \\
\tilde{\boldsymbol{X}}_{k_{\mid \mathcal{I}} \mid}
\end{array}\right]}_{\tilde{\boldsymbol{X}}} \\
+\underbrace{\left[\begin{array}{c}
\tilde{\boldsymbol{V}}_{k_{1}} \\
\vdots \\
\tilde{\boldsymbol{V}}_{k_{|\mathcal{I}+|}}
\end{array}\right]}_{\tilde{\boldsymbol{V}}}+\underbrace{\left[\begin{array}{c}
\tilde{\boldsymbol{N}}_{k_{1}} \\
\vdots \\
\tilde{\boldsymbol{N}}_{k_{|\mathcal{I}+|}}
\end{array}\right]}_{\tilde{\boldsymbol{N}}} .
\end{array}
$$

We first consider the capacity when there is no CFO and the IQ imbalance exists only on the transmitter side. Then, $\tilde{\boldsymbol{V}}=\mathbf{0}_{M N_{R} \times 1}$ in (38) and $\tilde{\boldsymbol{N}}$ in (38) is white ZMCSCG. For any given underlying channel realization $\boldsymbol{H}_{\text {total }} \triangleq$ $\left[\boldsymbol{H}^{(0)}, \ldots, \boldsymbol{H}^{\left(L_{1}-1\right)}\right]$, where $\boldsymbol{H}^{(l)} \triangleq\left\{h_{j i}^{(l)}\right\}_{j, i}$ (and, hence, a realization of $\tilde{\boldsymbol{H}}$ ), if $\mathcal{H}($.$) is the entropy function, the mutual$ information between input and output in (38) is given by

$$
\begin{aligned}
\mathcal{I}_{\mathrm{mi}}(\tilde{\boldsymbol{r}} ; \tilde{\boldsymbol{X}} \mid \tilde{\boldsymbol{H}}) & =\mathcal{H}(\tilde{\boldsymbol{r}} \mid \tilde{\boldsymbol{H}})-\mathcal{H}(\tilde{\boldsymbol{r}} \mid \tilde{\boldsymbol{X}}, \tilde{\boldsymbol{H}}) \\
& =\mathcal{H}(\tilde{\boldsymbol{r}} \mid \tilde{\boldsymbol{H}})-\mathcal{H}(\tilde{\boldsymbol{N}}) .
\end{aligned}
$$

The mutual information in (39) is maximized for circularly symmetric complex Gaussian input. In this case (i.e., for transmitter-side only IQ imbalance), we have $\mathcal{H}(\tilde{\boldsymbol{r}} \mid \tilde{\boldsymbol{X}}, \tilde{\boldsymbol{H}})=$ $\mathcal{H}(\tilde{\boldsymbol{N}})=\log \operatorname{det}\left(\pi e \sigma_{n}^{2} \boldsymbol{I}_{M N_{R}}\right)$, where $M \triangleq 2\left|\mathcal{I}^{+}\right|$is the total number of data tones. Hence, the mutual information in (39) is maximized if the entropy $\mathcal{H}(\tilde{\boldsymbol{r}} \mid \tilde{\boldsymbol{H}})$ is maximized. We know that for a given covariance matrix $\boldsymbol{\Sigma}_{\tilde{r}}$, a circularly symmetric complex Gaussian vector maximizes the entropy. Under a fixed channel realization $\boldsymbol{H}_{\text {total }}$, for circularly symmetric complex Gaussian input with covariance $\boldsymbol{\Sigma}_{\tilde{X}}$, the output is circularly symmetric complex Gaussian with covariance given by $\boldsymbol{\Sigma}_{\tilde{r}}=$ $\tilde{\boldsymbol{H}} \boldsymbol{\Sigma}_{\tilde{\boldsymbol{X}}} \tilde{\boldsymbol{H}}^{H}+N \sigma_{n}^{2} \boldsymbol{I}_{M N_{R}}$ and entropy given by $\mathcal{H}(\tilde{\boldsymbol{r}} \mid \tilde{\boldsymbol{H}})=$ $\log \operatorname{det}\left(\pi e \boldsymbol{\Sigma}_{\tilde{\boldsymbol{r}}}\right)$. Therefore

$$
\begin{aligned}
\mathcal{I}_{\text {mi }}\left(\tilde{\boldsymbol{r}} ; \tilde{\boldsymbol{X}} \mid \boldsymbol{H}_{\text {total }}\right) & =\log \operatorname{det}\left(\pi e \boldsymbol{\Sigma}_{\tilde{\boldsymbol{r}}}\right)-\log \operatorname{det}\left(\pi e N \sigma_{n}^{2} \boldsymbol{I}_{M N_{R}}\right) \\
& =\log \operatorname{det}\left(\boldsymbol{I}_{M N_{R}}+\frac{1}{N \sigma_{n}^{2}} \tilde{\boldsymbol{H}} \boldsymbol{\Sigma}_{\tilde{\boldsymbol{X}}} \tilde{\boldsymbol{H}}^{H}\right) .
\end{aligned}
$$

When the transmitter has no channel knowledge, the optimal power allocation is to allocate equal power among all input symbols over all tones [26]-[28]. Then, the input covariance is $\boldsymbol{\Sigma}_{\tilde{\boldsymbol{X}}}=P_{X} \boldsymbol{I}_{M N_{T}}$, and the ergodic capacity (normalized per OFDM subcarrier) becomes

$$
\begin{aligned}
C & =\frac{1}{\bar{N}} \mathrm{E}\left\{\mathcal{I}_{\mathrm{mi}}\right\} \\
& =\frac{1}{\bar{N}} \mathrm{E}\left[\log \left[\operatorname{det}\left(\boldsymbol{I}_{M N_{R}}+\frac{P_{X} \tilde{\boldsymbol{H}} \tilde{\boldsymbol{H}}^{H}}{N \sigma_{n}^{2}}\right)\right]\right] \\
& =\frac{1}{\bar{N}} \sum_{k \in \mathcal{I}^{+}} \mathrm{E}\left[\log \operatorname{det}\left(\boldsymbol{I}_{2 N_{R}}+\frac{P_{X}}{N \sigma_{n}^{2}} \boldsymbol{A}_{k}\right)\right]
\end{aligned}
$$

where

$$
\boldsymbol{A}_{k} \triangleq\left[\begin{array}{cc}
\boldsymbol{P}_{k} \boldsymbol{P}_{k}^{H}+\boldsymbol{Q}_{k} \boldsymbol{Q}_{k}^{H} & \boldsymbol{P}_{k} \boldsymbol{Q}_{-k}^{T}+\boldsymbol{Q}_{k} \boldsymbol{P}_{-k}^{T} \\
\boldsymbol{Q}_{-k}^{*} \boldsymbol{P}_{k}^{H}+\boldsymbol{P}_{-k}^{*} \boldsymbol{Q}_{k}^{H} & \boldsymbol{P}_{-k}^{*} \boldsymbol{P}_{-k}^{T}+\boldsymbol{Q}_{-k}^{*} \boldsymbol{Q}_{-k}^{T}
\end{array}\right] .
$$

$\bar{N} \triangleq N+L$ takes the overhead due to cyclic prefix into account, and the expectation is with respect to the underlying channel $\boldsymbol{H}_{\text {total }}$. Equation (41) is thus the ergodic capacity of MIMO-OFDM system with transmitter-side only IQ imbalance.

Next, we consider the presence of $\mathrm{CFO}$ and receive-side IQ imbalance. In this case, the effective noise $\tilde{\boldsymbol{V}}+\tilde{\boldsymbol{N}}$ is not circularly symmetric, and thus, it is not tractable to find the 
capacity, and we focus on finding a lower bound. Even to apply a lower bound as in [29], the model requires the effective noise to be uncorrelated with the data, whereas in this case, CFO results in correlation between the effective noise and data. However, on each direct-mirror tone pair, we have

$$
\mathrm{E}\left[\left[\begin{array}{cc}
\overline{\boldsymbol{X}}_{k}^{T} & \overline{\boldsymbol{X}}_{-k}^{H}
\end{array}\right]^{T}\left[\begin{array}{cc}
\tilde{\boldsymbol{V}}_{k}^{H} & \tilde{\boldsymbol{V}}_{-k}^{T}
\end{array}\right]=\mathbf{0}_{M N_{R} \times M N_{R}} .\right.
$$

The first term (and similarly the second term) on the diagonal block is zero since $\overline{\boldsymbol{V}}_{k}$ consists of all the tones except the $k$ th tone. The nondiagonal block terms are zero since the input is circularly symmetric. Thus, the CFO interference noise, and as a result the effective noise, on each direct-mirror tone pair is uncorrelated with the data signals. Hence, a lower bound on the capacity at each direct-mirror tone pair is obtained for independent circularly symmetric complex Gaussian noise with the same covariance as $\overline{\boldsymbol{D}}_{k} \triangleq \boldsymbol{R}_{\tilde{\boldsymbol{N}}_{k}}+\boldsymbol{R}_{\tilde{\boldsymbol{V}}_{k}}, \boldsymbol{R}_{\tilde{\boldsymbol{N}}_{k}} \triangleq \mathrm{E}\left[\tilde{\boldsymbol{N}}_{k} \tilde{\boldsymbol{N}}_{k}^{H}\right]$, and $\boldsymbol{R}_{\tilde{\boldsymbol{V}}_{k}} \triangleq \mathrm{E}\left[\tilde{\boldsymbol{V}}_{k} \tilde{\boldsymbol{V}}_{k}^{H}\right]$ and is given by

$$
C_{k} \geq \underline{\mathrm{C}}_{k} \triangleq \mathrm{E}\left[\log \operatorname{det}\left(\boldsymbol{I}_{N_{R}}+\overline{\boldsymbol{D}}_{k}^{-1} \tilde{\boldsymbol{H}}_{k} \tilde{\boldsymbol{H}}_{k}^{H}\right)\right] .
$$

The lower bound on the normalized sum capacity is then obtained by averaging the above capacity over all tones as

$$
\begin{aligned}
\underline{\mathrm{C}}^{\prime} & =\frac{1}{\bar{N}} \sum_{k \in \mathcal{I}^{+}} \underline{\mathrm{C}}_{k} \\
& =\frac{1}{\bar{N}} \sum_{k \in \mathcal{I}^{+}} \mathrm{E}\left[\log \operatorname{det}\left(\boldsymbol{I}_{N_{R}}+\overline{\boldsymbol{D}}_{k}^{-1} \tilde{\boldsymbol{H}}_{k} \tilde{\boldsymbol{H}}_{k}^{H}\right)\right] .
\end{aligned}
$$

The expression for $\boldsymbol{R}_{\tilde{\boldsymbol{V}}_{k}}$ is derived in the Appendix.

We now incorporate the channel estimation error into our model. From (27) and (28), using DFT, we obtain

$$
\hat{\boldsymbol{P}}_{k}=\boldsymbol{P}_{k}+\Delta \boldsymbol{P}_{k} \text { and } \hat{\boldsymbol{Q}}_{k}=\boldsymbol{Q}_{k}+\Delta \boldsymbol{Q}_{k}, \quad k \in \mathcal{I}
$$

where $\hat{\boldsymbol{P}}_{k} \triangleq\left\{\boldsymbol{f}_{k} \hat{\boldsymbol{p}}_{j i}\right\}_{j, i}, \Delta \boldsymbol{P}_{k} \triangleq\left\{\boldsymbol{f}_{k} \Delta \boldsymbol{p}_{j i}\right\}_{j, i}$ and $\Delta \boldsymbol{p}_{j i} \triangleq$ $\hat{\boldsymbol{p}}_{j i}-\boldsymbol{p}_{j i}$. $\hat{\boldsymbol{Q}}_{k}$, and $\Delta \boldsymbol{Q}_{k}$ are similarly defined. Using (46) in (38), we get

$$
\tilde{\boldsymbol{r}}_{k}=\hat{\tilde{\boldsymbol{H}}}_{k} \tilde{\boldsymbol{X}}_{k}-\Delta \tilde{\boldsymbol{H}}_{k} \tilde{\boldsymbol{X}}_{k}+\tilde{\boldsymbol{V}}_{k}+\tilde{\boldsymbol{N}}_{k}
$$

where $\hat{\tilde{\boldsymbol{H}}}_{k} \triangleq\left[\begin{array}{cc}\hat{\boldsymbol{P}}_{k} & \hat{\boldsymbol{Q}}_{k} \\ \hat{\boldsymbol{Q}}_{-k}^{*} & \hat{\boldsymbol{P}}_{-k}^{*}\end{array}\right]$, and $\Delta \tilde{\boldsymbol{H}}_{k} \triangleq\left[\begin{array}{cc}\Delta \boldsymbol{P}_{k} & \Delta \boldsymbol{Q}_{k} \\ \Delta \boldsymbol{Q}_{-k}^{*} & \Delta \boldsymbol{P}_{-k}^{*}\end{array}\right]$.
Here, the effective noise term $-\Delta \tilde{\boldsymbol{H}}_{k} \tilde{\boldsymbol{X}}_{k}+\tilde{\boldsymbol{V}}_{k}+\tilde{\boldsymbol{N}}_{k}$ is correlated with the channel estimate due to the LS estimate error term in the effective noise. However, at high SNR, this correlation is negligible, ${ }^{1}$ and hence, in the high SNR regime, we can find the lower bound on the normalized sum capacity over all tones as ${ }^{2}$

$$
C \geq \underline{\mathrm{C}} \triangleq \frac{1}{\bar{N}} \sum_{k \in \mathcal{I}^{+}} \mathrm{E}\left[\log \operatorname{det}\left(\boldsymbol{I}_{2 N_{R}}+\boldsymbol{D}_{k}^{-1} \hat{\boldsymbol{A}}_{k}\right)\right]
$$

where $\boldsymbol{D}_{k}$ and $\hat{\boldsymbol{A}}_{k}$ are expressed in (49), shown at the bottom of the page. In the above, we have used $P_{X}=\left(N P_{x} / M\right)$ assuming that $M$ out of $N$ subcarriers are used to transmit data. Evaluating the expectation, we have

$$
\boldsymbol{D}_{k}=\left[\begin{array}{cc}
a \boldsymbol{I}_{N_{R}} & \boldsymbol{\Lambda}_{\boldsymbol{b}^{k}} \\
\boldsymbol{\Lambda}_{\boldsymbol{b}^{k *}} & a \boldsymbol{I}_{N_{R}}
\end{array}\right]
$$

where $a=\left(2 L N_{T} \sigma_{n}^{2} / P_{s}\right)+\left(M \sigma_{n}^{2} / P_{x}\right), \boldsymbol{b}^{k}=\left[b_{1}^{k}, b_{2}^{k}, \ldots b_{N_{R}}^{k}\right]^{T}$, and $b_{j}^{k} \triangleq\left(2 / P_{s}^{2}\right) \boldsymbol{f}_{k}\left(\sum_{i=1}^{N_{T}} \boldsymbol{S}_{\mathrm{circ}, i}^{H} \boldsymbol{M}_{\boldsymbol{n}_{j}} \boldsymbol{S}_{\mathrm{circ}, i}\right) \boldsymbol{f}_{-k} \cdot{ }^{3}$

\section{Pilot-Data Power Allocation}

We define the ratio of the average data power to the total average power at each transmit antenna to be $\alpha$ with $0<\alpha<$ 1. Then, $P_{x}=\alpha P$ and $P_{s}=(1-\alpha) P$, where $P$ is the total average power in an OFDM symbol at each transmit antenna. For fixed $M$ and $K \triangleq N-M$, a closed-form optimal solution for $\alpha$ can be obtained for the high SNR regime, where we define SNR at the $j$ th receive antenna as $\mathrm{SNR}_{j}=\left(P \sum_{i=1}^{N_{T}}\left(\sigma_{P_{j i}}^{2}+\right.\right.$ $\left.\left.\sigma_{Q_{j i}}^{2}\right) / N \sigma_{n}^{2}\right), \sigma_{P_{j i}}^{2} \triangleq \sum_{l=0}^{L-1} \sigma_{p_{j i}^{(l)}}^{2}, \sigma_{Q_{j i}}^{2} \triangleq \sum_{l=0}^{L-1} \sigma_{q_{j i}^{(l)}}^{2}, \sigma_{p_{j i}^{(l)}}^{2}=$ $E\left[p_{j i}^{(l)} p_{j i}^{(l) *}\right]$, and $\sigma_{q_{j i}^{(l)}}^{2}=E\left[q_{j i}^{(l)} q_{j i}^{(l) *}\right]$. We also define the average receive $\mathrm{SNR}$ as $\mathrm{SNR}=\left(\sum_{j=1}^{N_{R}} \mathrm{SNR}_{j} / N_{R}\right)$. The pilot

\footnotetext{
${ }^{1}$ The correlation between estimate and error in the LS estimation is inversely proportional to SNR, and hence, we can approximate as uncorrelated in this regime. Alternatively, we can think of it in that at high SNR, the LS estimate is similar to the MMSE estimate with the property that the channel estimate and the estimation error are orthogonal.

${ }^{2}$ Nevertheless, this bound has been used in several works for maximum likelihood and LS estimates, e.g., [30] and [31], for all SNR regions, although it is accurate only in the high SNR regimes.

${ }^{3}$ In deriving the capacity bound in [1] for the SISO system, we neglected the nondiagonal terms, and therefore, the capacity bound expression in [1] can be seen as an approximation to the expression here after setting $N_{R}=N_{T}=1$. However, the pilot power allocation result for the SISO case in [1] remains the same as that derived for the MIMO case later in Section VI after setting $N_{T}=1$ in the MIMO power allocation result.
}

$$
\begin{aligned}
\boldsymbol{D}_{k} \triangleq & \mathrm{E}\left[\begin{array}{cc}
\Delta \boldsymbol{P}_{k} \Delta \boldsymbol{P}_{k}^{H}+\Delta \boldsymbol{Q}_{k} \Delta \boldsymbol{Q}_{k}^{H} & \Delta \boldsymbol{P}_{k} \Delta \boldsymbol{Q}_{-k}^{T}+\Delta \boldsymbol{Q}_{k} \Delta \boldsymbol{P}_{-k}^{T} \\
\Delta \boldsymbol{Q}_{-k}^{*} \Delta \boldsymbol{P}_{k}^{H}+\Delta \boldsymbol{P}_{-k}^{*} \Delta \boldsymbol{Q}_{k}^{H} & \Delta \boldsymbol{P}_{-k}^{*} \Delta \boldsymbol{P}_{-k}^{T}+\Delta \boldsymbol{Q}_{-k}^{*} \Delta \boldsymbol{Q}_{-k}^{T}
\end{array}\right] \\
& +\boldsymbol{R}_{\tilde{\boldsymbol{V}}_{k}}+\frac{M \sigma_{n}^{2}}{P_{x}} \boldsymbol{I}_{2 N_{R}}, \\
\hat{\boldsymbol{A}}_{k} \triangleq & {\left[\begin{array}{cc}
\hat{\boldsymbol{P}}_{k} \hat{\boldsymbol{P}}_{k}^{H}+\hat{\boldsymbol{Q}}_{k} \hat{\boldsymbol{Q}}_{k}^{H} & \hat{\boldsymbol{P}}_{k} \hat{\boldsymbol{Q}}_{-k}^{T}+\hat{\boldsymbol{Q}}_{k} \hat{\boldsymbol{P}}_{-k}^{T} \\
\hat{\boldsymbol{Q}}_{-k}^{*} \hat{\boldsymbol{P}}_{k}^{H}+\hat{\boldsymbol{P}}_{-k}^{*} \hat{\boldsymbol{Q}}_{k}^{H} & \hat{\boldsymbol{P}}_{-k}^{*} \hat{\boldsymbol{P}}_{-k}^{T}+\hat{\boldsymbol{Q}}_{-k}^{*} \hat{\boldsymbol{Q}}_{-k}^{T}
\end{array}\right] }
\end{aligned}
$$


data power allocation problem to maximize the capacity lower bound is stated as

$$
\alpha_{\mathrm{opt}}=\arg \max _{\alpha} \underline{\mathrm{C}}, \quad 0 \leq \alpha \leq 1 .
$$

At high SNR, the channel estimation is nearly perfect, and hence, $\hat{\boldsymbol{P}}_{k} \hat{\boldsymbol{P}}_{k}^{H}+\hat{\boldsymbol{Q}}_{k} \hat{\boldsymbol{Q}}_{k}^{H} \approx \boldsymbol{P}_{k} \boldsymbol{P}_{k}^{H}+\boldsymbol{Q}_{k} \boldsymbol{Q}_{k}^{H}$. Similarly, the other terms in (49) can be simplified. Therefore, in the high SNR regime, we can approximate (48) as

$$
\underline{\mathrm{C}} \approx \frac{1}{\bar{N}} \sum_{k \in \mathcal{I}^{+}} \mathrm{E}\left[\log \left(\operatorname{det}\left[\boldsymbol{A}_{k}\right]\right)\right]+\frac{1}{\bar{N}} \sum_{k \in \mathcal{I}^{+}} \log \left(\operatorname{det}\left[\boldsymbol{D}_{k}^{-1}\right]\right) .
$$

The power allocation factor $\alpha$ affects (52) only through the term $(1 / \bar{N}) \sum_{k \in \mathcal{I}} \log \left(\operatorname{det}\left[\boldsymbol{D}_{k}^{-1}\right]\right)$, and hence, $\underline{C}$ is maximized by maximizing it. However, the term $\boldsymbol{D}_{k}$ and hence the optimizing power allocation depend on the CFO, which is not known a priori at the transmitter, and hence, we design the power allocation neglecting the CFO, and we will study how the CFO changes the power allocation factor using the exact expression in the simulations.

Therefore, the optimal $\alpha$ for the high SNR regime is given by

$$
\begin{aligned}
\alpha_{\text {opt,highSNR }} & =\arg \max _{\alpha} \frac{1}{\bar{N}} \sum_{k \in \mathcal{I}} \log \left(\operatorname{det}\left[\boldsymbol{D}_{k}^{-1}\right]\right) \\
& =\arg \min _{\alpha} \sum_{k \in \mathcal{I}} \Pi_{j=1}^{N_{R}}\left(a^{2}-\left|b_{j}^{k}\right|^{2}\right)
\end{aligned}
$$

where we used the determinant and inverse of a block matrix in simplifying above. Optimizing the foregoing expression requires knowledge of the IQ parameters (which appear in $b_{j}^{k}$ terms), which is not available at the transmitter. However, for all practical IQ imbalances, we have $a^{2} \gg\left|b_{j}^{k}\right|^{2}$ due to $a$ consisting of noise covariance terms and $b_{j}^{k}$ consisting of noise pseudocovariance terms. Hence, using (13), we get

$$
a^{2}-\left|b_{j}^{k}\right|^{2} \approx a^{2}
$$

and the optimization problem can be solved as

$$
\begin{aligned}
\alpha_{\text {opt,highSNR }} & =\arg \min _{\alpha} a=\arg \min _{\alpha} \frac{2 L N_{T}}{(1-\alpha) P}+\frac{M}{\alpha P} \\
& =\frac{1}{1+\sqrt{\frac{2 L N_{T}}{M}}} .
\end{aligned}
$$

\section{SLOW FADing Channels}

In this section, we consider a slow fading channel and assume it remains static over several OFDM symbols, for example, $Q$ symbols. This is when we have the case $Q T_{s} \ll T_{c}$, where $T_{s}$ and $T_{c}$ are the OFDM symbol time and the channel coherence time, respectively. In such a case, only the first OFDM symbol needs to contain pilots, whereas the remaining symbols contain data only. The pilot-data power allocation can be derived using a similar approach.

\section{A. Equal Data Tone Power Across All OFDM Symbols}

The lower bound on the total capacity for the $q$ th OFDM symbol is then given by

$$
\underline{\mathrm{C}}_{q} \triangleq \frac{1}{\bar{N}} \sum_{k_{q} \in \mathcal{I}_{q}^{+}} \mathrm{E}\left[\log \operatorname{det}\left(\boldsymbol{I}_{2 N_{R}}+\boldsymbol{D}_{k_{q}}^{-1} \hat{\boldsymbol{A}}_{k_{q}}\right)\right]
$$

where $\mathcal{I}_{q}^{+}$is the set of direct data tones in the $q$ th OFDM symbol. We also define $M_{q}=\left|\mathcal{I}_{q}\right|$ as the number of elements in $\mathcal{I}_{q}$. The lower bound on the average capacity per tone will then be

$$
\underline{\mathrm{C}}_{\mathrm{avg}}=\frac{1}{Q \bar{N}} \sum_{q=1}^{Q} \underline{\mathrm{C}}_{q}
$$

We can see that at high SNR, the power allocation affects the capacity bound only through the $\boldsymbol{D}_{k_{q}}$ terms, which, under approximation (55), are equal for all the symbols. Hence, we optimize (58) for power allocation. Let $P_{x_{q}}$ be the data power allocation for the $q$ th OFDM symbol and $P_{s}+\sum_{q=1}^{Q} P_{x_{q}}=$ $P_{\text {tot }}$ be the total power constraint on the block of $Q$ OFDM symbols. Then, we need to minimize $\left(2 L N_{T} / P_{s}\right)+\left(N / P_{X}\right)$ under the total power constraint and $P_{x_{q}}=\left(M_{q} P_{X} / N\right)$. Solving this optimization problem, the pilot and data power allocations are obtained as follows:

$$
\begin{aligned}
P_{s} & =\frac{\sqrt{2 L N_{T}} P_{\mathrm{tot}}}{\left(\sqrt{M_{\mathrm{sum}}}+\sqrt{2 L N_{T}}\right)} \\
P_{x_{1}} & =\frac{M_{1} P_{\mathrm{tot}}}{\sqrt{M_{\mathrm{sum}}}\left(\sqrt{M_{\mathrm{sum}}}+\sqrt{2 L N_{T}}\right)} \\
P_{x_{q}} & =\frac{M_{q} P_{\mathrm{tot}}}{\sqrt{M_{\mathrm{sum}}}\left(\sqrt{M_{\mathrm{sum}}}+\sqrt{2 L N_{T}}\right)}
\end{aligned}
$$

where $M_{\text {sum }} \triangleq \sum_{q=1}^{Q} M_{q}$.

\section{B. Large Number of Symbols Per Block}

This is the case of slowly varying channels when we can have a large number of symbols per block, i.e., $Q \gg 1$. In this case, (58) is approximated as $\underline{C}_{\mathrm{avg}} \approx(1 / Q \bar{N}) \sum_{k=2}^{Q} \underline{C}_{k}$. Consider the particular case of equal number of data tones in each of the $Q-1$ OFDM symbols. Let $M_{2}$ and $P_{2}$ denote the number of data tones and data power allocation, respectively, in each of the $Q-1$ OFDM symbols. The total power constraint is then $P_{x_{1}}+P_{s_{1}}+(Q-1) P_{x_{2}} \approx P_{s_{1}}+Q P_{x_{2}}=$ $P_{\text {tot }}$. The power allocation factor is defined as $Q P_{x_{2}}=$ $\alpha P_{\text {tot }}$ and $P_{s_{1}}=(1-\alpha) P_{\text {tot }}$. Therefore, the factor to be minimized is $\gamma=\left(2 L N_{T} / P_{s_{1}}\right)+\left(M_{2} / P_{x_{2}}\right)=\left(2 L N_{T} /(1-\right.$ $\left.\alpha) P_{\text {tot }}\right)+\left(Q M_{2} / \alpha P_{\text {tot }}\right)$, solving which yields the optimal power allocation as

$$
\alpha_{\mathrm{opt}, \text { largeQ }}=\frac{1}{1+\sqrt{\left(\frac{2 L N_{T}}{M_{2} Q}\right)}} .
$$




\section{General Case}

In this section, we relax the assumptions in the foregoing two sections and consider the power allocation for any general $Q$ and flexible data tone powers across the $Q$ OFDM symbols. At high SNR, we can write (58) as

$$
\begin{aligned}
\underline{\mathrm{C}}_{\mathrm{avg}}= & \frac{1}{\bar{N} Q} \sum_{q=1}^{Q} \sum_{k \in \mathcal{I}_{q}}\left(\log \operatorname{det}\left(\boldsymbol{D}_{k_{q}}^{-1}\right)+\mathrm{E}\left[\log \operatorname{det}\left(\boldsymbol{A}_{k_{q}}\right)\right]\right) \\
= & {\left[\log \operatorname{det}\left(\Pi_{q=1}^{Q} \Pi_{k \in \mathcal{I}_{q}}\left(\boldsymbol{D}_{k_{q}}\right)^{\frac{-1}{N Q}}\right)\right] } \\
& +\mathrm{E}\left[\log \operatorname{det}\left(\Pi_{q=1}^{Q} \Pi_{k \in \mathcal{I}_{q}}\left(\boldsymbol{A}_{k_{q}}\right)^{\frac{1}{N Q}}\right)\right]
\end{aligned}
$$

Using (55), the optimal power allocation is then obtained by minimizing $\Pi_{q=1}^{Q}\left(\left(2 L N_{T} \sigma_{n}^{2} / P_{s}\right)+\left(M_{q} \sigma_{n}^{2} / P_{x_{k}}\right)\right)^{\left(M_{q} / \bar{N} Q\right)}$ under the constraint $P_{s}+\sum_{q=1}^{Q} P_{x_{q}}=P_{\text {tot }}$. Therefore, if we let $P_{x_{q}}=\alpha_{q} P_{\text {tot }}, q=1,2, \ldots, Q$, and $P_{s}=\beta P_{\text {tot }}$, where $\beta \triangleq 1-\sum_{q=1}^{Q} \alpha_{q}$, the optimal $\alpha_{q}$ 's and $\beta$ are given by

$$
\begin{aligned}
& \left\{\alpha_{1, \mathrm{opt}}, \ldots, \alpha_{Q, \mathrm{opt}}, \beta_{\mathrm{opt}}\right\} \\
& \quad=\arg \min _{\alpha_{1}, \ldots, \alpha_{Q}, \beta} \Pi_{q=1}^{Q}\left(\frac{2 L N_{T}}{\beta}+\frac{M_{q}}{\alpha_{q}}\right)^{\frac{M_{q}}{N Q}}
\end{aligned}
$$

under the constraint $0 \leq \alpha_{q} \leq 1$ for $q=1,2, \ldots, Q, 0 \leq \beta \leq$ 1 , and $0 \leq \beta+\sum_{q=1}^{Q} \alpha_{q} \leq 1$. A solution to the above can be obtained by a numerical method where a grid search over discrete values of $\alpha_{q}$ 's and $\beta$ is performed within the range [0, 1] that minimizes the preceding expression. As an example, for $N=64, N_{T}=2, L=8, Q=4, M_{1}=30$, and $M_{2}=M_{3}=$ $M_{4}=62$, we get the power allocation parameters as $\alpha_{1}=0.12$, $\beta=0.28$, and $\alpha_{2}=\alpha_{3}=\alpha_{4}=0.20$.

\section{PERFORMANCE COMPARISON}

We use a $2 \times 3$ MIMO-OFDM system with $N=64$ subcarriers for our simulation. We set $L_{1}=4, Z=3$, and $L=L_{1}+$ $2 Z-2=8$. We use $K \triangleq 2 L=16$ pilot tones at each transmit antenna and $M=N-2 L N_{T}-2=30$ data tones within the pilot-data multiplexed OFDM symbol. In all the simulations, quaternary phase-shift keying was used. For the spatial correlation, we used $\boldsymbol{R}_{R}(j, i)=\rho_{R}^{|i-j|}$ and $\boldsymbol{R}_{T}(j, i)=\rho_{T}^{|i-j|}$, with $\rho_{R}=0.4$ and $\rho_{T}=0.7$. We assumed an exponential power delay profile for the underlying channel.

In Figs. 2 and 3, we study the effect of IQ imbalance and CFO on capacity bound and SER under perfect channel state information (CSI) at the receiver. In the legend, IQ1, IQ2, and IQ3 represent increasing severities of IQ imbalance frequency selectivity. Their detailed descriptions are given in the caption of Fig. 2. While IQ1 represents typical IQ imbalance seen in practical systems, IQ2 and IQ3 represent much higher severity than what is typically observed in practice. From Fig. 2, we see that for any given CFO, with increasing IQ imbalance frequency selectivity, the capacity bound reduces. This can be attributed to the increased correlation and channel fluctuations produced by higher frequency-selective IQ imbalance. Next, we notice that as CFO is increased, the capacity bound reduces.

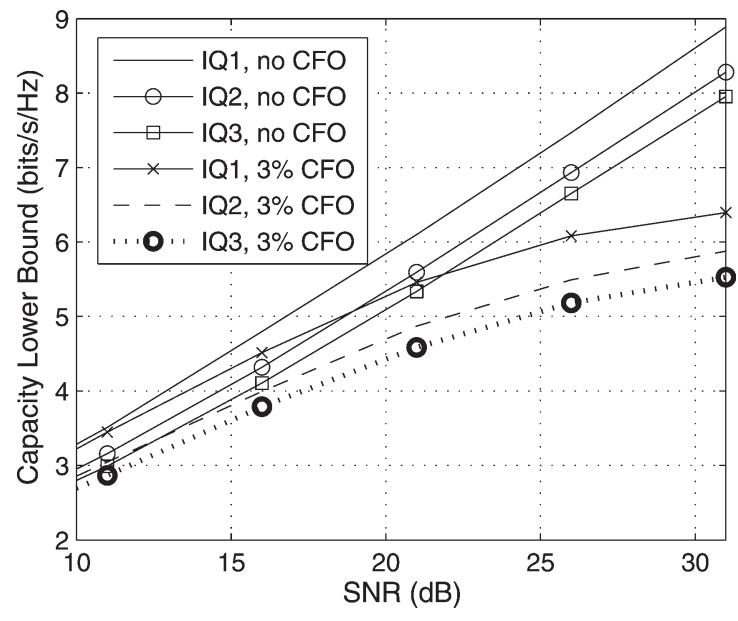

Fig. 2. Effect of frequency-selective IQ imbalance and CFO on capacity bound for $N_{T}=2, N_{R}=3$ MIMO-OFDM with perfect CSI at receiver and fast fading. The CFO percentages are with respect to the OFDM subcarrier spacing.IQ 1: $\boldsymbol{g}_{t, i}^{I}=[0.01,0.9999,0.01], \boldsymbol{g}_{t, i}^{Q}=[0.015,0.9998,0.01], \boldsymbol{g}_{r, j}^{I}=$ $[0.012,0.9997,0.018], \quad \boldsymbol{g}_{r, j}^{Q}=[0.01,0.9997,0.02] a_{t, i}^{I}=1.0227, \quad a_{t, i}^{Q}=$ 0.9767, $a_{r, j}^{I}=1.0227, a_{r, j}^{Q}=0.9767, \theta_{t, i}^{I}=4^{\circ}, \theta_{t, i}^{Q}=0, \theta_{r, j}^{I}=4^{\circ}$, $\theta_{r, j}^{Q}=0 . \mathrm{IQ} \quad 2: \quad \boldsymbol{g}_{t, i}^{I}=[0.2236,0.9487,0.2236], \quad \boldsymbol{g}_{t, i}^{Q}=[0.2,0.9592,0.2]$, $\boldsymbol{g}_{r, j}^{I}=[0.2646,0.9434,0.2], \boldsymbol{g}_{r, j}^{Q}=[0.2236,0.922,0.3162], a_{t, i}^{I}=1.0488$, $a_{t, i}^{Q}=0.9487, a_{r, j}^{I}=1.0724, a_{r, j}^{Q}=0.922, \theta_{t, i}^{I}=4^{\circ}, \theta_{t, i}^{Q}=3, \theta_{r, j}^{I}=4^{\circ}$, $\theta_{r, j}^{Q}=2$. IQ 3: $\boldsymbol{g}_{t, i}^{I}=[0.2236,0.8944,0.3873], \boldsymbol{g}_{t, i}^{Q}=[0.2,0.9055,0.3742]$, $\boldsymbol{g}_{r, j}^{I}=[0.2,0.9434,0.2646], \quad \boldsymbol{g}_{r, j}^{Q}=[0.1414,0.9055,0.4] a_{t, i}^{I}=1.1402$, $a_{t, i}^{Q}=0.8367, \quad a_{r, j}^{I}=1.1180, \quad a_{r, j}^{Q}=0.8660, \quad \theta_{t, i}^{I}=5^{\circ}, \quad \theta_{t, i}^{Q}=4$, $\theta_{r, j}^{I}=6^{\circ}, \theta_{r, j}^{Q}=2$.

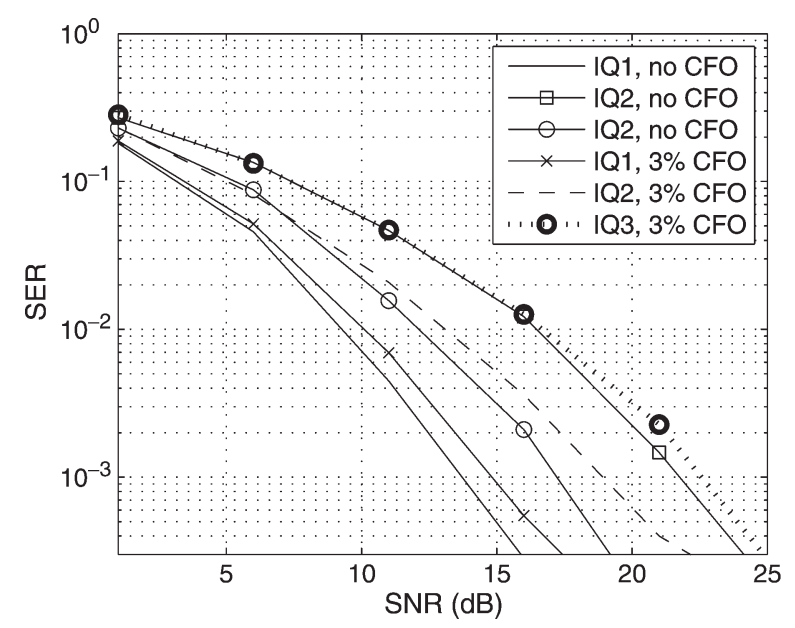

Fig. 3. Effect of frequency-selective IQ imbalance and CFO on SER for $N_{T}=2, N_{R}=3$ MIMO-OFDM with perfect CSI at receiver and fast fading.

This is due to the greater interference produced with higher CFO. In addition, we notice that for the $3 \% \mathrm{CFO}$ case (percentage here is in terms of subcarrier spacing), the increase in capacity with SNR at higher SNR regions is much slower. This is due to the fact that as SNR is increased, the signal and interference powers are proportional, and hence, the gain in capacity comes from reduced noise variance, which is close to negligible at high SNR regimes. We further notice that for the considered cases of IQ severities and CFO, the capacity is more sensitive to $\mathrm{CFO}$ than to IQ imbalance. 


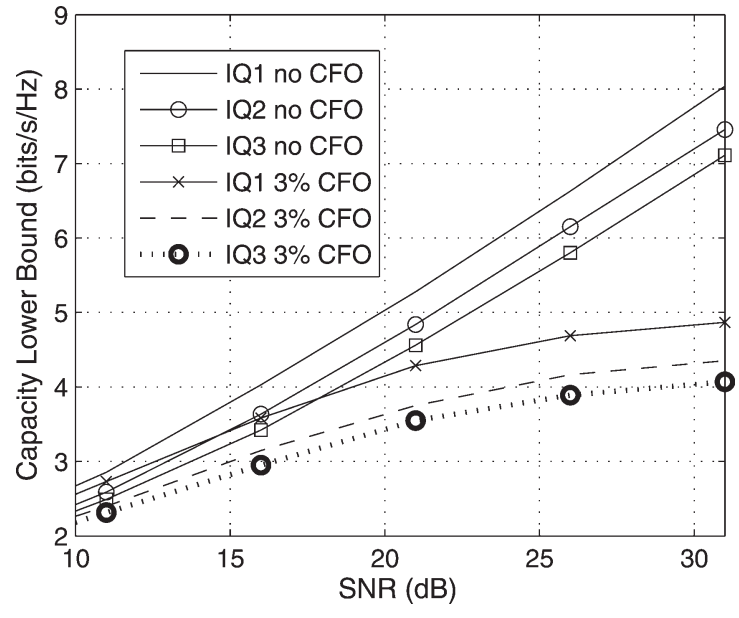

Fig. 4. Effect of frequency-selective IQ imbalance and CFO on capacity bound for $N_{T}=2, N_{R}=3$ MIMO-OFDM with imperfect CSI at receiver and fast fading.

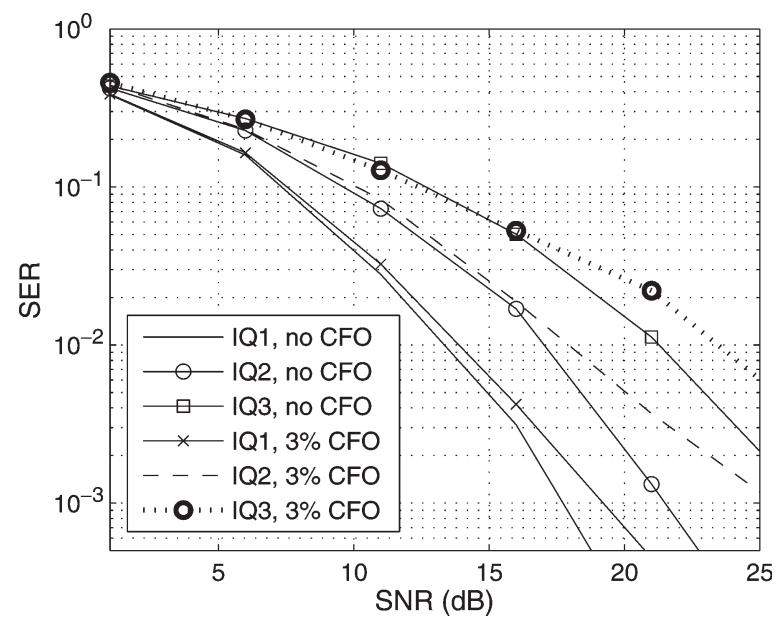

Fig. 5. Effect of frequency-selective IQ imbalance and CFO on SER for $N_{T}=2, N_{R}=3$ MIMO-OFDM with imperfect CSI at receiver and fast fading.

Fig. 3 presents the SER results for different IQ imbalance cases and CFO under perfect CSI. First, we observe that both increased IQ imbalance severity and CFO degrade SER performance. However, unlike the capacity results, we notice that SER is more adversely affected by IQ imbalance severity than by CFO. Figs. 4 and 5 show the capacity bound and SER incorporating channel estimation at the receiver. The same general trend as in Figs. 2 and 3 is observed here, though the capacity and SER at any point are much worse than the corresponding points in Figs. 2 and 3 due to the channel estimation errors.

In Figs. 6 and 7, we study the effect of pilot-data power allocation on capacity and SER at SNR $=15 \mathrm{~dB}$. We see that the maximum capacity is very close to the derived power allocation of $\alpha_{\text {opt,highSNR }}=\left(1 / 1+\sqrt{\left(2 L N_{T} / M\right)}\right)=0.492$ for the no CFO case and for increasing CFO cases up to $3 \%$ CFO. We observe that the capacity is not very sensitive to power allocation factor around the peak value and even power allocation changes up to about 0.1 , i.e., $20 \%$ on either side of the optimal power allocation yield very close to the maximum

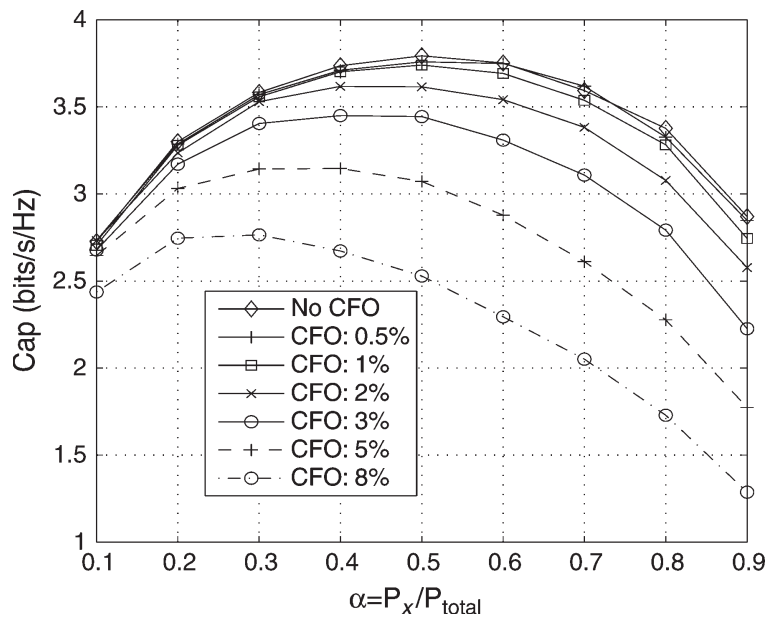

Fig. 6. Effect of power allocation on capacity bound for various CFO severities at SNR $=15 \mathrm{~dB}$ and IQ1 (parameters: $M=30, N_{t}=2, N_{R}=3$, $N=64)$.

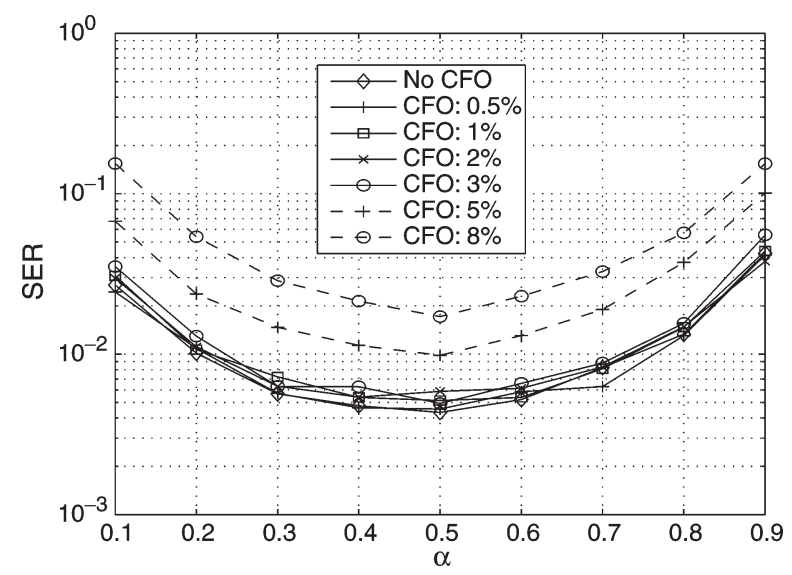

Fig. 7. Effect of power allocation on SER for various CFO severities at $\mathrm{SNR}=15 \mathrm{~dB}$ and IQ1 (parameters: $M=30, N_{t}=2, N_{R}=3, N=64$ ).

capacity. Beyond this change in the power allocation, we can see a noticeable decrease in capacity. For CFO values beyond $3 \%$, the optimizing power allocation shifts to the left, i.e., the maximum capacity occurs for less data and more pilot power allocation. This is due to the fact that for increasing CFO, higher data power allocation results in the interference playing a dominant role in lowering the capacity. For 5\% CFO, we see about 3\% decrease in capacity at our derived power allocation compared with the peak in this case.

From Fig. 7, we notice a different effect of the power allocation factor on the SER compared with its effect on capacity. While increasing CFO degrades SER, we notice that the minimum of the SER curves did not significantly shift for all the considered CFO values. This can also be explained from the results of Figs. 3 and 5, where we noticed that SER is not particularly sensitive to CFO in the considered range of CFO values. While SER performance is not directly related to capacity, interestingly, the power allocation at which the minimum SER occurs in all the CFO ranges closely matches our derived power allocation.

In Fig. 8, we study the effect of power allocation factor and CFO on capacity bound at higher SNR of $20 \mathrm{~dB}$. In this case and 


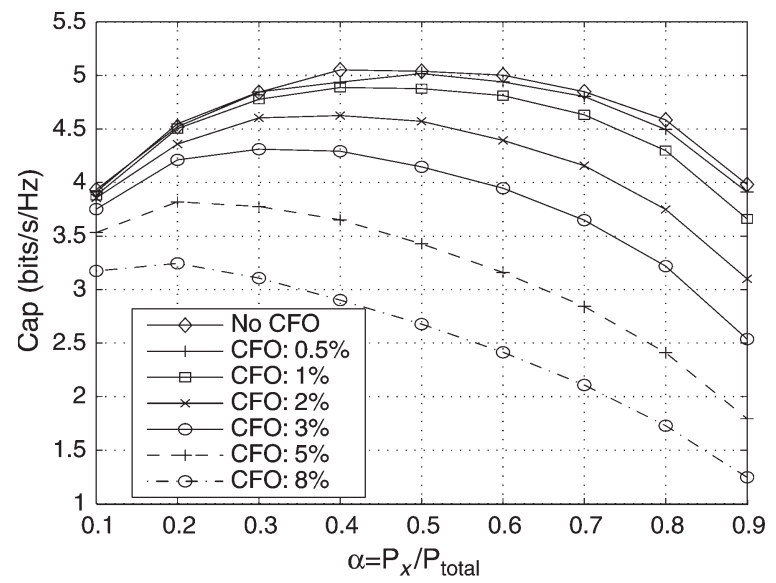

Fig. 8. Effect of power allocation on capacity bound for various $\mathrm{CFO}$ severities at SNR $=20 \mathrm{~dB}$ and IQ1 (parameters: $M=30, N_{t}=2, N_{R}=3$, $N=64)$.

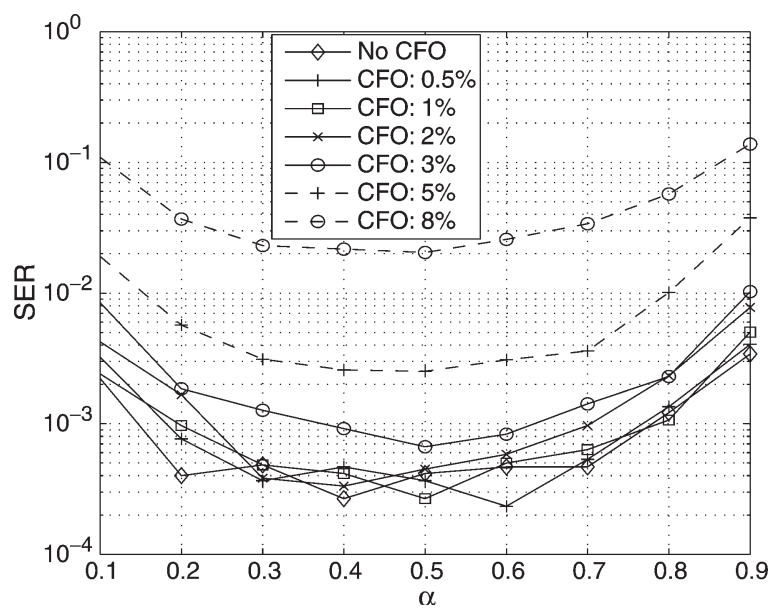

Fig. 9. Effect of power allocation on SER for various CFO severities at SNR $=20 \mathrm{~dB}$ and IQ1 (parameters: $M=30, N_{t}=2, N_{R}=3, N=64$ ).

with increasing SNRs in general, we observe that significant changes in peak capacity compared with the no CFO case start occurring at lower $\mathrm{CFO}$ values. For example, at $15 \mathrm{~dB}$, while $3 \% \mathrm{CFO}$ had peak at the derived power allocation, at $20 \mathrm{~dB}$, we notice a shift in maximizing power allocation by about 0.2 (i.e., $40 \%$ ), and the maximum capacity is off by about $2.5 \%$ from the capacity at the derived power allocation. As SNR is further increased, we notice that the capacity maximizing power allocation shifts to the left (i.e., suggesting less power be allocated to data). However, from the SER curves in Figs. 7 and 9, SER is close to minimum at the derived power allocation even for high values of residual CFO. Therefore, considering only a slight loss in capacity at the derived power allocation compared with the peak, but almost the same SER performance, in addition to the fact that residual CFO is usually very small, the derived power allocation offers a viable solution for practical systems with IQ imbalance and residual CFO. Similar results were also obtained for the slow fading case and for uniform power delay profile of the underlying channel case. ${ }^{4}$

\footnotetext{
${ }^{4}$ Not shown here due to page limitations.
}

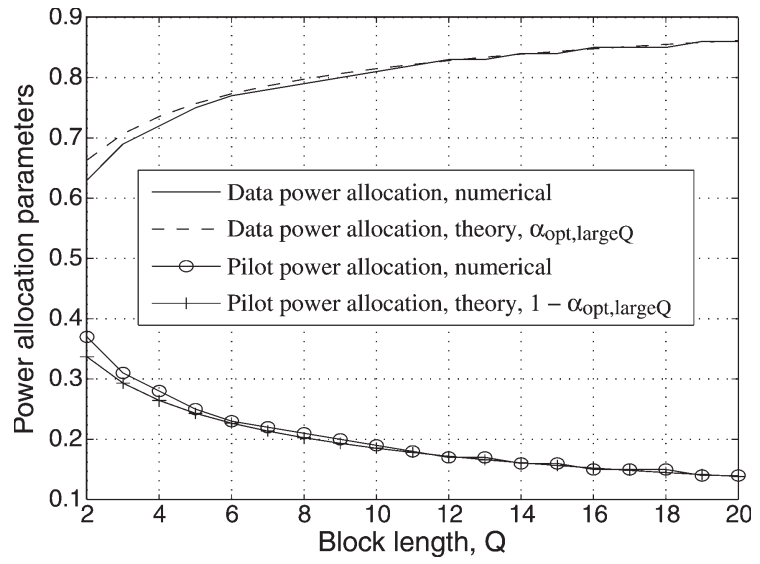

Fig. 10. Comparison of power allocation parameters obtained from the numerical method and the analytical result for a slow fading channel (parameters: $\left.M_{1}=30, M_{2}=\cdots=M_{Q}=62, N_{t}=2, N=64\right)$.

Considering the foregoing results, we learn that capacity bound is very sensitive to CFO, showing severe degradation, but for a given CFO, it is not very sensitive to the maximizing power allocation. On the other hand, SER is very sensitive to IQ imbalance frequency selectivity, although it is highly insensitive to the minimizing pilot-data power allocation. Thus, while the system must be designed to keep these impairments small, a wide choice of pilot-data power allocations around the derived power allocation is possible to obtain near best performance for the given IQ and CFO impairments.

Fig. 10 compares the optimal power allocation parameters for the slow fading case obtained using numerical evaluation in case $\mathrm{C}$ of Section VI and the analytical result $\alpha_{\mathrm{opt}, \text { largeQ }}$ for large $Q$. We see that for $Q>6$, the data and pilot powers predicted by the large $Q$ approximation is quite close to the exact powers obtained from the numerical evaluation. In addition, results from the numerical evaluation suggest that the data power allocation has to be divided among the individual OFDM symbols in proportion to the number of data tones in the individual symbols, which is the same as having equal data tone power across all the OFDM symbols. Case A of Section VI gives the closed-form result for this case, and hence, it can be used to obtain the power allocation for any general $Q$ and $M_{q}$ for $q=1,2, \ldots, Q$.

\section{CONCLUSION}

We have analyzed the ergodic capacity of MIMO-OFDM suffering from frequency-selective IQ imbalances at the transmit and receive sides and residual CFO. The derived capacity expression is the capacity of the system when the IQ imbalance exists only on the transmit side without CFO. For IQ imbalances at both the transmit and receive sides along with $\mathrm{CFO}$ and imperfect channel estimation at the receiver, we derived a lower bound on the capacity. We found a closed-form pilotdata power allocation that maximizes the capacity bound under no CFO assumption and studied how the CFO affects the maximizing power allocation. We noticed that both capacity and SER degrade with increasing IQ imbalance and CFO severity. 
However, capacity was more sensitive to CFO, whereas SER was more sensitive to IQ imbalance severity. In the presence of CFO, when either CFO or SNR was increased, the capacity was maximized for lower power allocated to data. Despite capacity and SER showing high sensitivity to CFO and IQ imbalance frequency selectivity, we observed that neither is very sensitive to the optimizing power allocation, e.g., even for moderately high residual CFO of 3\%, the maximum capacity and the capacity at the derived power allocation matched closely at $15 \mathrm{~dB}$ and were off by about $2.5 \%$ at $20 \mathrm{~dB}$, whereas SER remained close to the minimum at the derived power allocation. These results suggest that from the capacity and SER perspective, the derived power allocation is a good choice for low and even moderately high CFO while a wide choice of power allocations exist that attain near best performance for the given IQ imbalance and CFO severity.

\section{APPENDIX}

Proof of Number of Taps in Effective Direct and Mirror Channels: Consider $\boldsymbol{P}_{\text {circ }, \boldsymbol{j i}}$ from (23). The number of nonzero elements in the first column of $\boldsymbol{P}_{\text {circ }, j i}$ is the number of channel taps in the effective direct channel. Neglecting the constant $1 / N$, the first column is given by $\left\{\boldsymbol{P}_{\text {circ }, \boldsymbol{j} i}\right\}_{j, 1}$

$$
\begin{aligned}
= & \boldsymbol{F}^{H} \operatorname{diag}\left(\boldsymbol{A}_{1, j i}\right) \boldsymbol{f}_{1}^{T}+\boldsymbol{F}^{H} \operatorname{diag}\left(\boldsymbol{A}_{2, j i}\right) \boldsymbol{f}_{1}^{T} \\
= & \boldsymbol{F}^{H}\left[\boldsymbol{A}_{1, j i}(1,1), \boldsymbol{A}_{1, j i}(2,2), \ldots, \boldsymbol{A}_{1, j i}(N, N)\right]^{T} \\
& +\boldsymbol{F}^{H}\left[\boldsymbol{A}_{2, j i}(1,1), \boldsymbol{A}_{2, j i}(2,2), \ldots, \boldsymbol{A}_{2, j i}(N, N)\right]^{T} .
\end{aligned}
$$

From (15), we know that

$$
\begin{aligned}
& \boldsymbol{A}_{1, j i}(k, k)=\boldsymbol{f}_{k} \boldsymbol{G}_{R, j}^{D} \boldsymbol{W}(\nu) \boldsymbol{G}_{D, i}^{T} \boldsymbol{H}_{j i} \tilde{P} \boldsymbol{f}_{k}^{H} \\
& \boldsymbol{A}_{2, j i}(k, k)=\boldsymbol{f}_{k} \boldsymbol{G}_{R, j}^{M} \boldsymbol{W}^{*}(\nu) \boldsymbol{G}_{M, i}^{T *} \boldsymbol{H}_{j i}^{*} \tilde{P} \boldsymbol{f}_{k}^{H} .
\end{aligned}
$$

Since $\boldsymbol{G}_{R, j}^{D}, \boldsymbol{G}_{D, i}^{T}$, and $\boldsymbol{H}_{j i}$ are convolution matrices with $Z, Z$, and $L_{1}$ taps, respectively, $\boldsymbol{W}(\nu)$ is diagonal, and we know the structure of the permutation matrix $\widetilde{\boldsymbol{P}}$, we can verify that the matrices $\overline{\boldsymbol{C}}_{1} \triangleq \boldsymbol{G}_{R, j}^{D} \boldsymbol{W}(\nu) \boldsymbol{G}_{D, i}^{T} \boldsymbol{H}_{j i} \tilde{P}$ and $\overline{\boldsymbol{C}}_{2} \triangleq$ $\boldsymbol{G}_{R, j}^{M} \boldsymbol{W}^{*}(\nu) \boldsymbol{G}_{M, i}^{T *} \boldsymbol{H}_{j i}^{*} \tilde{P}$ have the form that the $i$ th column has nonzero elements in the $i$ to $(i+L-1)_{N}$ rows, where the subscript indicates modulo $N$. We represent the $i$ th column in $\overline{\boldsymbol{C}}_{1}$ and $\overline{\boldsymbol{C}}_{2}$ by $c_{1, i}$ and $c_{2, i}$, respectively. Thus, (66) can be written as

$$
\begin{aligned}
& \boldsymbol{A}_{1, j i}(k, k)=\sum_{i=1}^{N} \boldsymbol{f}_{k}^{*}(1, i) \boldsymbol{f}_{k} \boldsymbol{c}_{1, i} \\
& \boldsymbol{A}_{2, j i}(k, k)=\sum_{i=1}^{N} \boldsymbol{f}_{k}^{*}(1, i) \boldsymbol{f}_{k} \boldsymbol{c}_{2, i} .
\end{aligned}
$$

Substituting this into (65), we can write the $l$ th element of the first column of $\boldsymbol{P}_{\text {circ }, j i}$ as $\boldsymbol{P}_{\text {circ }, j i}(l, 1)$ in (70) and (71), shown at the bottom of the page, where $d_{m} \triangleq \sum_{i=1}^{N}\left(c_{1, i}(i+m, 1)+\right.$ $\left.c_{2, i}(i+m, 1)\right)$. From the above, we see that the coefficient of each $d_{m}, m=0, \ldots, L-1$, is zero except when $l=m$. Thus, for $l=0,1, \ldots, L-1$, there is one nonzero term in the summation, and hence, the corresponding element in the first column of $\boldsymbol{P}_{\text {circ }, j i}$ is nonzero. For $l>L-1$, we see that all the coefficients in the summation are zero, and hence, we conclude that the first column of $\boldsymbol{P}_{\text {circ }, j i}$ has only $L$ nonzero elements. We know that this is the number of taps in the equivalent time-domain direct channel. Using the same argument, we can show that the time-domain mirror channel has a maximum of $L$ taps.

$$
\begin{aligned}
&=\sum_{k=0}^{N-1} e^{j \frac{2 \pi k l}{N}}\left(\sum _ { i = 0 } ^ { N - 1 } e ^ { j \frac { j 2 \pi k i } { N } } \left(\sum_{m=i}^{(i+L-1)_{N}} e^{-j \frac{2 \pi k m}{N}}\right.\right.\left.\left.\times\left(c_{1, i}(m, 1)+c_{2, i}(m, 1)\right)\right)\right) \\
&=\sum_{k=0}^{N-1} e^{j \frac{2 \pi k l}{N}}\left(\sum _ { i = 0 } ^ { N - 1 } \left(e^{-0}\left(c_{1, i}(i, 1)+c_{2, i}(i, 1)\right)\right.\right. \\
&+e^{-j \frac{2 \pi k}{N}}\left(c_{1, i}(i+1,1)+c_{2, i}(i+1,1)\right)+\ldots \\
&\left.\left.+e^{-j \frac{2 \pi k(L-1)}{N}}\left(c_{1, i}(i+L-1,1)+c_{2, i}(i+L-1,1)\right)\right)\right) \\
&=\sum_{m=0}^{L-1} d_{m} \sum_{k=0}^{N-1} e^{j \frac{2 \pi k(l-m)}{N}}=\sum_{m=0}^{L-1} d_{m} \delta[l-m]
\end{aligned}
$$


Algorithm for Finding Correlations Involving Interferences: To find the interference correlations in time and frequency domain and, hence, evaluate the effect of residual CFO and IQ imbalance on channel capacity, we will need expressions for $\boldsymbol{R}_{k 1, k 2} \triangleq E\left[\boldsymbol{V}_{k_{1}} \boldsymbol{V}_{k_{2}}^{H}\right]$ and $\overline{\boldsymbol{R}}_{k 1, k 2} \triangleq E\left[\boldsymbol{V}_{k_{1}} \boldsymbol{V}_{k_{2}}^{T}\right]$, from which all the other correlation expressions can be obtained. Consider

$$
\begin{aligned}
\boldsymbol{R}_{k_{1}, k_{2}}=E[ & {\left[\begin{array}{lll}
{\left[\sum_{i=1}^{N_{T}} V_{k_{1}, 1 i}\right.} & \ldots & \sum_{i=1}^{N_{T}} V_{k_{1}, N_{R} i}
\end{array}\right]^{T} } \\
& \times\left[\begin{array}{llll}
\sum_{i=1}^{N_{T}} V_{k_{2}, 1 i}^{H} & \ldots & \sum_{i=1}^{N_{T}} V_{k_{2}, N_{R} i}^{H}
\end{array}\right]
\end{aligned}
$$

where

$$
\begin{aligned}
& V_{k, j i} \triangleq \boldsymbol{f}_{k} \bar{A}_{1, j i} \boldsymbol{H}_{j i} \tilde{\boldsymbol{P}} \boldsymbol{F}^{H} \boldsymbol{X}_{i}^{(k)}+\boldsymbol{f}_{k} \bar{A}_{2, j i} \boldsymbol{H}_{j i}^{*} \tilde{\boldsymbol{P}} \boldsymbol{F}^{H} \boldsymbol{X}_{i}^{(k)} \\
& +\boldsymbol{f}_{k} \bar{B}_{1, j i} \boldsymbol{H}_{j i} \tilde{\boldsymbol{P}} \boldsymbol{F}^{H} \boldsymbol{X}_{-, i}^{(-k) *}+\boldsymbol{f}_{k} \bar{B}_{2, j i} \boldsymbol{H}_{j i}^{*} \tilde{\boldsymbol{P}} \boldsymbol{F}^{H} \boldsymbol{X}_{-, i}^{(-k) *} .
\end{aligned}
$$

$V_{k, j i}$ is the $k$ th tone interference term at the $j$ th receive antenna coming from the $i$ th transmit antenna, and we have used the notation $\boldsymbol{X}_{i}^{(k)}$ to denote vector $\boldsymbol{X}_{i}$ with a zero in the $k$ th tone location. To compute the above, we need to compute $E\left[V_{k, j i} V_{k, l m}^{H}\right]$. For $i \neq m$, since the data at different transmit antennas are independent of each other with zero mean, we have $E\left[V_{k_{1}, j i} V_{k_{2}, l m}^{H}\right]=0$. Therefore, we consider the case for $i=m$. In this case, we have

$$
\begin{aligned}
E & {\left[V_{k_{1}, j i} V_{k_{2}, l i}^{H}\right] } \\
& =E\left[\boldsymbol{f}_{k_{1}} \overline{\boldsymbol{A}}_{1, j i} \boldsymbol{H}_{j i} \tilde{\boldsymbol{P}} \boldsymbol{F}^{H} \boldsymbol{C}_{\boldsymbol{X}_{i}^{\left(k_{1}, k_{2}\right)}} \boldsymbol{F} \tilde{\boldsymbol{P}}^{H} \boldsymbol{H}_{l i}^{H} \boldsymbol{A}_{1, l i}^{H} \boldsymbol{f}_{k_{2}}^{H}\right] \\
& +E\left[\boldsymbol{f}_{k_{1}} \overline{\boldsymbol{A}}_{2, j i} \boldsymbol{H}_{j i}^{*} \tilde{\boldsymbol{P}} \boldsymbol{F}^{H} \boldsymbol{C}_{\boldsymbol{X}_{i}^{\left(k_{1}, k_{2}\right)}} \boldsymbol{F} \tilde{\boldsymbol{P}}^{H} \boldsymbol{H}_{l i}^{T} \boldsymbol{A}_{2, l i} \boldsymbol{f}_{k_{2}}^{H}\right] \\
& +E\left[\boldsymbol{f}_{k_{1}} \overline{\boldsymbol{B}}_{1, j i} \boldsymbol{H}_{j i} \tilde{\boldsymbol{P}} \boldsymbol{F}^{H} \boldsymbol{C}_{\boldsymbol{X}_{-, i}^{\left(-k_{1},-k_{2}\right) *}} \boldsymbol{F} \tilde{\boldsymbol{P}}^{H} \boldsymbol{H}_{l i}^{H} \boldsymbol{B}_{1, l i}^{H} \boldsymbol{f}_{k_{2}}^{H}\right] \\
& +E\left[\boldsymbol{f}_{k_{1}} \overline{\boldsymbol{B}}_{2, j i} \boldsymbol{H}_{j i}^{*} \tilde{\boldsymbol{P}} \boldsymbol{F}^{H} \boldsymbol{C}_{\boldsymbol{X}_{-, i}^{\left(-k_{1}, k_{2}\right) *}} \boldsymbol{F} \tilde{\boldsymbol{P}}^{H} \boldsymbol{H}_{l i}^{T} \boldsymbol{B}_{2, l i}^{H} \boldsymbol{f}_{k_{2}}^{H}\right] \\
& =\boldsymbol{f}_{k_{1}} \overline{\boldsymbol{A}}_{1, j i} \boldsymbol{W}_{j, l, i}^{\left(k_{1}, k_{2}\right)} \overline{\boldsymbol{A}}_{1, j i}^{H} \boldsymbol{f}_{k_{2}}^{H}+\boldsymbol{f}_{k_{1}} \overline{\boldsymbol{A}}_{2, j i} \boldsymbol{W}_{j, l, i}^{\left(k_{1}, k_{2}\right)} \overline{\boldsymbol{A}}_{2, j i}^{H} \boldsymbol{f}_{k_{2}}^{H} \\
& +\boldsymbol{f}_{k_{1}} \overline{\boldsymbol{B}}_{1, j i} \boldsymbol{W}_{j, l, i}^{\left(k_{1}, k_{2}\right)} \overline{\boldsymbol{B}}_{1, j i}^{H} \boldsymbol{f}_{k_{2}}^{H}+\boldsymbol{f}_{k_{1}} \overline{\boldsymbol{B}}_{2, j i} \boldsymbol{W}_{j, l, i}^{\left(k_{1}, k_{2}\right)} \overline{\boldsymbol{B}}_{2, j i}^{H} \boldsymbol{f}_{k_{2}}^{H}
\end{aligned}
$$

where

$$
\begin{aligned}
\boldsymbol{C}_{\boldsymbol{X}_{i}^{\left(k_{1}, k_{2}\right)}} \triangleq E\left[\boldsymbol{X}_{i}^{\left(k_{1}\right)} \boldsymbol{X}_{i}^{\left(k_{2}\right) H}\right]=\boldsymbol{C}_{\boldsymbol{X}_{-, i}^{\left(-k_{1},-k_{2}\right) *}} \\
\boldsymbol{W}_{j, l, i}^{\left(k_{1}, k_{2}\right)} \triangleq\left\{\boldsymbol{W}_{j, l, i}^{\left(k_{1}, k_{2}\right)}(x, y)\right\}_{x, y=1, \ldots, N+2 Z-2} \\
\boldsymbol{W}_{j, l, i}^{\left(k_{1}, k_{2}\right)}(x, y) \triangleq \rho_{R}^{|j-l|} \sum_{m=0}^{L-1} \gamma_{m} \boldsymbol{U}_{i}^{\left(k_{1}, k_{2}\right)}(x+m, y+m) \\
\boldsymbol{U}_{i}^{\left(k_{1}, k_{2}\right)} \triangleq \tilde{\boldsymbol{P}} \boldsymbol{F}^{H} \boldsymbol{C}_{\boldsymbol{X}_{i}^{\left(k_{1}, k_{2}\right)}} \boldsymbol{F} \tilde{\boldsymbol{P}}^{H}
\end{aligned}
$$

$\gamma_{l} \triangleq \mathrm{E}\left[\left|h_{j i}^{(l)}\right|^{2}\right]$ is the power in the $l$ th tap, and $\rho_{R}^{|j-i|}$ is the correlation coefficient between $j$ th and $i$ th receive antennas.
Using (74) in (72), we get

$$
\begin{aligned}
\boldsymbol{R}_{k_{1}, k_{2}}= & \left\{\sum _ { i = 1 } ^ { N _ { T } } \left(\boldsymbol{f}_{k_{1}} \overline{\boldsymbol{A}}_{1, j i} \boldsymbol{W}_{j, l, i}^{\left(k_{1}, k_{2}\right)} \overline{\boldsymbol{A}}_{1, j i}^{H} \boldsymbol{f}_{k_{2}}^{H}\right.\right. \\
& \left.\left.+\boldsymbol{f}_{k_{1}} \overline{\boldsymbol{A}}_{2, j i} \boldsymbol{W}_{j, l, i}^{\left(k_{1}, k_{2}\right)} \overline{\boldsymbol{A}}_{2, j i}^{H} \boldsymbol{f}_{k_{2}}^{H}\right)\right\}_{j, l=1, \ldots, N_{R}} \\
& +\left\{\sum _ { i = 1 } ^ { N _ { T } } \left(\boldsymbol{f}_{k_{1}} \overline{\boldsymbol{B}}_{1, j i} \boldsymbol{W}_{j, l, i}^{\left(k_{1}, k_{2}\right)} \overline{\boldsymbol{B}}_{1, j i}^{H} \boldsymbol{f}_{k_{2}}^{H}\right.\right. \\
& \left.\left.+\boldsymbol{f}_{k_{1}} \overline{\boldsymbol{B}}_{2, j i} \boldsymbol{W}_{j, l, i}^{\left(k_{1}, k_{2}\right)} \overline{\boldsymbol{B}}_{2, j i}^{H} \boldsymbol{f}_{k_{2}}^{H}\right)\right\}_{j, l=1, \ldots, N_{R}}
\end{aligned}
$$

Using a similar approach, we obtain

$$
\begin{aligned}
& \overline{\boldsymbol{R}}_{k_{1}, k_{2}}=\left\{\sum _ { i = 1 } ^ { N _ { T } } \left(\boldsymbol{f}_{k_{1}} \overline{\boldsymbol{A}}_{1, j i} \tilde{\boldsymbol{W}}_{j, l, i}^{\left(k_{1}, k_{2}\right)} \overline{\boldsymbol{B}}_{2, j i}^{T} \boldsymbol{f}_{k_{2}}^{*}\right.\right. \\
&\left.\left.+\boldsymbol{f}_{k_{1}} \overline{\boldsymbol{A}}_{2, j i} \tilde{\boldsymbol{W}}_{j, l, i}^{\left(k_{1}, k_{2}\right)} \overline{\boldsymbol{B}}_{1, j i}^{T} \boldsymbol{f}_{k_{2}}^{*}\right)\right\}_{j, l=1, \ldots, N_{R}} \\
&+\left\{\sum _ { i = 1 } ^ { N _ { T } } \left(\boldsymbol{f}_{k_{1}} \overline{\boldsymbol{B}}_{1, j i} \tilde{\boldsymbol{W}}_{j, l, i}^{\left(k_{1}, k_{2}\right)} \overline{\boldsymbol{A}}_{2, j i}^{T} \boldsymbol{f}_{k_{2}}^{*}\right.\right. \\
&\left.\left.+\boldsymbol{f}_{k_{1}} \overline{\boldsymbol{B}}_{2, j i} \tilde{\boldsymbol{W}}_{j, l, i}^{\left(k_{1}, k_{2}\right)} \overline{\boldsymbol{A}}_{1, j i}^{T} \boldsymbol{f}_{k_{2}}^{*}\right)\right\}_{j, l=1, \ldots, N_{R}}
\end{aligned}
$$

where

$$
\begin{aligned}
\tilde{\boldsymbol{W}}_{j, l, i}^{\left(k_{1}, k_{2}\right)} & \triangleq\left\{\tilde{\boldsymbol{W}}_{j, l, i}^{k_{1}, k_{2}}(x, y)\right\}_{x, y=1, \ldots, N+2 Z-2} \\
\tilde{\boldsymbol{W}}_{j, l, i}^{\left(k_{1}, k_{2}\right)}(x, y) & \triangleq \rho_{R}^{|j-l|} \sum_{m=0}^{L-1} \gamma_{m} \tilde{\boldsymbol{U}}_{i}^{k_{1}, k_{2}}(x+m, y+m) \\
\tilde{\boldsymbol{U}}_{i}^{k_{1}, k_{2}} & \triangleq \tilde{\boldsymbol{P}} \boldsymbol{F}^{H} \tilde{\boldsymbol{C}}_{\boldsymbol{X}_{i}^{\left(k_{1}, k_{2}\right)} \boldsymbol{F}^{T} \tilde{\boldsymbol{P}}^{T}} \\
\tilde{\boldsymbol{C}}_{\boldsymbol{X}_{i}^{\left(k_{1}, k_{2}\right)}} & \triangleq E\left[\boldsymbol{X}_{i}^{\left(k_{1}\right)} \boldsymbol{X}_{-, i}^{\left(-k_{2}\right) H}\right] .
\end{aligned}
$$

Using (76) and (77), we can obtain

$$
\begin{aligned}
\boldsymbol{R}_{\tilde{\boldsymbol{V}}_{k}} & \triangleq\left[\begin{array}{cc}
\boldsymbol{R}_{k, k} & \overline{\boldsymbol{R}}_{k,-k} \\
\overline{\boldsymbol{R}}_{k,-k}^{H} & \boldsymbol{R}_{-k,-k}^{*}
\end{array}\right] \\
\boldsymbol{R}_{\boldsymbol{v}_{j}} & =E\left[\boldsymbol{v}_{j} \boldsymbol{v}_{j}^{H}\right] \\
& =\frac{1}{N^{2}}\left\{\boldsymbol{F}^{H} \boldsymbol{R}_{k_{1}, k_{2}}(j, j) \boldsymbol{F}\right\}_{k_{1}, k_{2}=\left\{\{1, \ldots, N-1\} \backslash\left\{\frac{N}{2}\right\}\right\}} \\
\overline{\boldsymbol{R}}_{\boldsymbol{v}_{j}} & =E\left[\boldsymbol{v}_{j} \boldsymbol{v}_{j}^{T}\right] \\
& =\frac{1}{N^{2}}\left\{\boldsymbol{F}^{H} \overline{\boldsymbol{R}}_{k_{1}, k_{2}}(j, j) \boldsymbol{F}^{*}\right\}_{k_{1}, k_{2}=\left\{\{1, \ldots, N-1\} \backslash\left\{\frac{N}{2}\right\}\right\}} .
\end{aligned}
$$




\section{REFERENCES}

[1] V. K. V. Gottumukkala and H. Minn, "Optimal pilot power allocation for OFDM systems with transmitter and receiver IQ imbalances," in Proc. IEEE GLOBECOM, 2009, pp. 1-5.

[2] S. Krone and G. Fettweis, "On the capacity of OFDM systems with receiver I/Q imbalance," in Proc. ICC, 2008, pp. 1317-1321.

[3] S. Krone and G. Fettweis, "Capacity analysis for OFDM systems with transceiver I/Q imbalance," in Proc. GLOBECOM, 2008, pp. 1-6.

[4] M. Krondorf and G. Fettweis, "OFDM link performance analysis under various receiver impairments," EURASIP J. Wireless Commun. Netw., vol. 2008, pp. 145 279-1-145 279-11, 2008.

[5] J. Qi and S. Aïssa, "On the effect of I/Q imbalance on MIMO transmitreceive diversity systems," in Proc. WCNC, 2009, pp. 1-5.

[6] T. Yoo and A. Goldsmith, "Capacity and power allocation for fading MIMO channels with channel estimation error," IEEE Trans. Inf. Theory, vol. 52, no. 5, pp. 2203-2214, May 2006.

[7] W. Kirkland and K. Teo, "I/Q distortion correction for OFDM direct conversion receiver," Electron. Lett., vol. 39, no. 1, pp. 131-133, Jan. 2003.

[8] Y. Egashira, Y. Tanabe, and K. Sato, "A novel IQ imbalance compensation method with pilot-signals for OFDM system," in Proc. IEEE VTC, Fall 2006, pp. 1-5.

[9] A. Tarighat, R. Bagheri, and A. H. Sayed, "Compensation schemes and performance analysis of IQ imbalances in OFDM receivers," IEEE Trans. Signal Process., vol. 53, no. 8, pp. 3257-3268, Aug. 2005.

[10] R. Chrabieh and S. Soliman, "IQ imbalance mitigation via unbiased training sequences," in Proc. IEEE GLOBECOM, 2007, pp. 4280-4285.

[11] E. Lopez-Estraviz, S. De Rore, F. Horlin, and A. Bourdoux, "Pilot design for joint channel and frequency-dependent transmit/receive IQ imbalance estimation and compensation in OFDM-based transceivers," in Proc IEEE ICC, 2007, pp. 4861-4866.

[12] T. Schenk, P. Smulders, and E. Fledderus, "Estimation and compensation of TX and RX IQ imbalance in OFDM based MIMO systems," in Proc. IEEE RWS, 2006, pp. 215-218.

[13] H. Minn and D. Munoz, "Pilot designs for channel estimation of MIMO OFDM systems with frequency-dependent I/Q imbalances," IEEE Trans. Commun., vol. 58, no. 8, pp. 2252-2264, Aug. 2010.

[14] J. Yu, M. Sun, T. Hsu, and C. Lee, "A novel technique for I/Q imbalance and cfo compensation in OFDM systems," in Proc. IEEE Int. Symp. Circuits Syst., 2005, pp. 6030-6033.

[15] B. Narasimhan, D. Wang, S. Narayanan, H. Minn, and N. Al-Dhahir, "Digital compensation of frequency-dependent joint Tx/Rx I/Q imbalance in OFDM systems under high mobility," IEEE J. Sel. Topics Signal Process., vol. 3, no. 3, pp. 405-417, Jun. 2009.

[16] M. Valkama, M. Renfors, and V. Koivunen, "Compensation of frequencyselective I/Q imbalances in wideband receivers: Models and algorithms," in Proc IEEE 3rd Workshop SPAWC, Mar. 20-23, 2001, pp. 42-45.

[17] J. Tubbax, A. Fort, L. Van der Perre, S. Donnay, M. Engels, M. Moonen, and H. De Man, "Joint compensation of IQ imbalance and frequency offset in OFDM systems," in Proc. IEEE GLOBECOM, 2003, vol. 4, pp. 2365-2369.

[18] D. Tandur and M. Moonen, "Joint adaptive compensation of transmitter and receiver iq imbalance under carrier frequency offset in OFDM-based systems," IEEE Trans. Signal Process., vol. 55, no. 11, pp. 5246-5252, Nov. 2007.

[19] J. Feigin and D. Brady, "Joint transmitter/receiver I/Q imbalance compensation for direct conversion OFDM in packet-switched multipath environments," IEEE Trans. Signal Process., vol. 57, no. 11, pp. 4588-4593, Nov. 2009.

[20] L. He, S. Ma, Y. Wu, Y. Zhou, T. Ng, and H. Poor, "Pilot-aided iq imbalance compensation for OFDM systems operating over doubly selective channels," IEEE Trans. Signal Process., vol. 59, no. 5, pp. 2223-2233, May 2011

[21] F. Lopez-Martinez, E. Martos-Naya, J. Paris, and J. Entrambasaguas, "Exact closed-form BER analysis of OFDM systems in the presence of IQ imbalances and ICSI," IEEE Trans. Wireless Commun., vol. 10, no. 6, pp. 1914-1922, Jun. 2011.
[22] S. De Rore, E. Lopez-Estraviz, F. Horlin, and L. Van der Perre, "Joint estimation of carrier frequency offset and iq imbalance for $4 \mathrm{~g}$ mobile wireless systems," in Proc. ICC, 2006, vol. 5, pp. 2066-2071.

[23] F. Horlin, A. Bourdoux, and L. Van der Perre, "Low-complexity EMbased joint acquisition of the carrier frequency offset and IQ imbalance," IEEE Trans. Wireless Commun., vol. 7, no. 6, pp. 2212-2220, Jun. 2008.

[24] I. Barhumi and M. Moonen, "IQ-imbalance compensation for OFDM in the presence of IBI and carrier-frequency offset," IEEE Trans. Signal Process., vol. 55, no. 1, pp. 256-266, Jan. 2007.

[25] Y. Chung and S. Phoong, "Joint estimation of I/Q imbalance, CFO and channel response for mimo OFDM systems," IEEE Trans. Commun., vol. 58, no. 5, pp. 1485-1492, May 2010.

[26] E. Telatar, "Capacity of multi-antenna Gaussian channels," Eur Trans. Telecommun., vol. 10, no. 6, pp. 585-595, Dec. 1999.

[27] G. Raleigh and J. Cioffi, "Spatio-temporal coding for wireless communication," IEEE Trans. Commun., vol. 46, no. 3, pp. 357-366, Mar. 2002.

[28] G. Raleigh and V. Jones, "Multivariate modulation and coding for wireless communication," IEEE J. Sel. Areas Comm., vol. 17, no. 5, pp. 851-866, May 2002.

[29] B. Hassibi and B. Hochwald, "How much training is needed in multipleantenna wireless links?" IEEE Trans. Inf. Theory, vol. 49, no. 4, pp. $951-$ 963, Apr. 2003.

[30] C. Pirak, Z. Wang, K. Liu, and S. Jitapunkul, "Optimum power allocation for maximum-likelihood channel estimation in space-time coded mimo systems," in Proc. ICASSP, 2006, vol. 4, p. IV.

[31] X. Liu, M. Bialkowski, and F. Wang, "Investigations into the effect of spatial correlation on channel estimation and capacity of multiple input multiple output system," Int. J. Commun. Netw. Syst. Sci., vol. 2, no. 4, pp. 267-275, Jul. 2009.

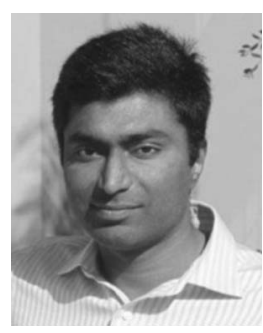

V. K. Varma Gottumukkala received the B.Tech. degree from the Indian Institute of Technology Delhi, New Delhi, India, and the Masters degree in electrical engineering from Texas A\&M University, College Station. He is currently working toward the Ph.D. degree in electrical engineering with the University of Texas at Dallas, Richardson.

His research interests are in the broad areas of wireless communications, signal processing, and networking.

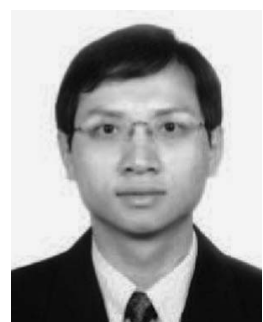

Hlaing Minn (S'99-M'01-SM'07) received the B.E. degree in electronics from the Yangon Institute of Technology, Yangon, Myanmar, in 1995, the M.Eng. degree in telecommunications from the Asian Institute of Technology, Pathumthani, Thailand, in 1997, and the Ph.D. degree in electrical engineering from the University of Victoria, Victoria, BC, Canada, in 2001.

During January-August 2002, he was a PostDoctoral Fellow with the University of Victoria. Since September 2002, he has been with the Erik Jonsson School of Engineering and Computer Science, University of Texas at Dallas, Richardson, where he is currently an Associate Professor. His research interests include wireless communications, statistical signal processing, error control, detection, estimation, synchronization, signal design, cross-layer design, cognitive radios, and wireless health-care applications.

Mr. Minn is an Editor for the IEEE TRANSACTIONS ON CommunicaTIONS and the International Journal of Communications and Networks. He served as Co-Chair of the Wireless Access Track in the IEEE Vehicular Technology Conference 2009 (Fall). 